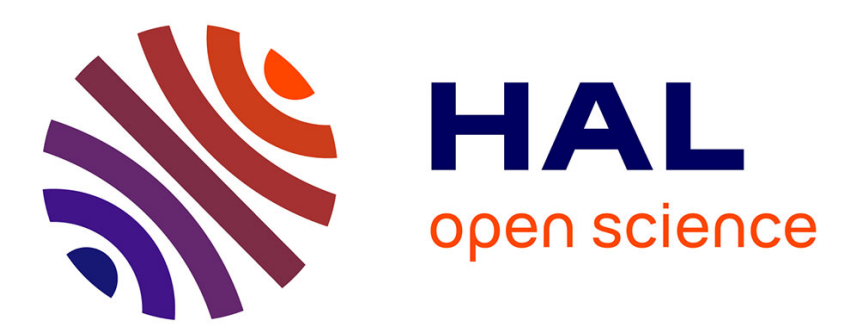

\title{
Controverses judéo-chrétiennes et iconographie. L'apport des inscriptions
}

Robert Favreau

\section{To cite this version:}

Robert Favreau. Controverses judéo-chrétiennes et iconographie. L'apport des inscriptions. Comptesrendus des séances de l'Académie des inscriptions et belles-lettres, 2001, 145 (3), pp.1267-1303. 10.3406/crai.2001.16339 . halshs-03206353

\section{HAL Id: halshs-03206353 \\ https://shs.hal.science/halshs-03206353}

Submitted on 23 Apr 2021

HAL is a multi-disciplinary open access archive for the deposit and dissemination of scientific research documents, whether they are published or not. The documents may come from teaching and research institutions in France or abroad, or from public or private research centers.
L'archive ouverte pluridisciplinaire HAL, est destinée au dépôt et à la diffusion de documents scientifiques de niveau recherche, publiés ou non, émanant des établissements d'enseignement et de recherche français ou étrangers, des laboratoires publics ou privés. 


\section{Controverses judéo-chrétiennes et iconographie. L'apport des} inscriptions

\section{Robert Favreau}

\section{Citer ce document / Cite this document :}

Favreau Robert. Controverses judéo-chrétiennes et iconographie. L'apport des inscriptions. In: Comptes rendus des séances de l'Académie des Inscriptions et Belles-Lettres, 145 e année, N. 3, 2001. pp. 1267-1303;

doi : https://doi.org/10.3406/crai.2001.16339

https://www.persee.fr/doc/crai_0065-0536_2001_num_145_3_16339

Fichier pdf généré le 19/07/2018 


\title{
COMMUNICATION
}

\author{
CONTROVERSES JUDEO-CHRETIENNES ET ICONOGRAPHIE. \\ L'APPORT DES INSCRIPTIONS, \\ PAR M. ROBIERT FAVREAI , CORRESPONDANT DE LACADÉMIE
}

Le problème des rapports entre judaïsme et christianisme est trop souvent du seul domaine des historiens du judaïsme. Les prendre en compte est nécessaire pour l'historien et l'historien de l'art du Moyen Âge, tant ces rapports ont été continus, et à certaines époques soutenus, au point qu'il faut se demander s'ils n'ont pas eu des répercussions dans l'iconographie du Moyen Âge. Bernhard Blumenkranz a abordé le problème à plusieurs reprises. Il souligne que dans la seule littérature chrétienne des époques patristique et médiévale une bonne centaine de textes ont pour seule fonction la polémique anti-juive. Il évoque une des " disputes " les plus importantes pour le sujet, celle de Gilbert Crispin et d'un Juif de Mayence. Il note comme participant à la polémique anti-juive la formulation de la doctrine d'après laquelle tout le Nouveau Testament est annoncé par l'Ancien Testament. Il s'intéresse à la représentation de la Synagogue et de façon plus générale de Juifs dans l'art médiéval, notamment dans les Bibles moralisées ${ }^{1}$. Récemment Laurence Brugger a montré que le cycle de la Genèse à la cathédrale de Bourges avait des sources importantes dans la littérature juive ${ }^{2}$. Étudiant la « soudaine résurgence

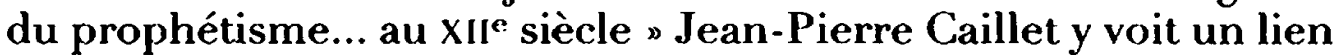
avec a l'exacerbation de l'anti-judaïsme... Les chrétiens veulent montrer que les juifs n'ont pas su lire dans l'Ancien Testament les signes annonciateurs du Messie... C'est incontestablement au

1. B. Blumenkranz, I La polémique: anti-juive dans l'art chrétien du Moyen Âge:Bollettino dell'Istimuo storico italiano per il Medio Evo e Archivio muratoriano) 77, 196:), p. 21.43; Id., - La représentation de Synagoga dans les Bibles moralisées françaises du VIII" au $\mathrm{KI}^{*}$ siecoles ", Proceedings of the Israel Academy of Sciences and IIumanities V/2, 1970, p. 70-91 : Id., Jee Juif médiéval au mircir de l'art chrélien, Paris, 1966. Voir aussi : S. I.jpton. Images of Intolerance : The Representation of Jews and Judaism in the Bible Moralisée, loss Angreles, 1999.

2. "llebraei dicum : le soubassement de la façade occidentale de la cathédrale de: Bourges ". Cahiers archéologiques 11, 1993, p. 111-139: La façade de Saint-Étienne de Bourges. Le Midrash comme fondement du message chétien (Givilisation médiévale. IX). Poitiers. 2000, thèse de doctorat de l'T'niversite de Geneve (1999). 


\section{COMPTES RENDUS DE I'ACADÉMIE DES INSCRIPTIONS}

XII* siècle que les œuvres d'art, reflet de leur temps, témoignent d'une prédilection très nette pour cette thématique $n^{3}$. Je voudrais, pour ma part, utiliser les inscriptions, qui nous renseignent directement sur les intentions des auteurs des programmes, pour apporter une contribution supplémentaire à l'étude de l'influence des controverses judéo-chrétiennes dans l'iconographie du Moyen Âge.

Les discussions religieuses entre juifs et chrétiens remontent aux origines mêmes de l'Église. L'épître de Paul aux Galates est ainsi une réponse aux judaïsants qui voudraient imposer aux païens convertis la pratique de la loi de Moïse et notamment la circoncision. Tertullien écrit au début du III" siècle son Adversus Judaeos à propos d'une discussion entre un chrétien et un prosélyte juif ; Clément d'Alexandrie à la même époque, répond dans ses Stromates ou Tapisseries aux objections des Grecs et des Juifs et Origène, son disciple, parle de discussions qu'il a eues avec des rabbins en présence d'une foule; saint Justin a, lui, écrit un Dialogue avec le Juif Tryphon à la suite d'une discussion, à Éphèse, avec un Juif célèbre de son temps. A Évagre on doit une Altercatio Theophili christiani et Simonis judaei, à Augustin un Tractatus adversus Judaeos, à Maxime de Turin un traité intitulé lui aussi " contre les Juifs ", et parmi les auteurs qui ont traité de la question juive il faut encore citer, pour l'Espagne, Isidore de Séville, Ildefonse de Tolède, Julien de Tolède, Paul Alvare de Cordoue. Pour le IX* siècle on a les évêques de Lyon Agobard et Amolon et l'archevêque de Mayence Raban Maur ${ }^{\prime \prime}$, pour le Xe siècle une Altercatio Aecclesie contra Synagogam et Synagoge contra Aecclesiam d'un auteur anonyme 5 . Ces noms, auxquels d'autres pourraient être ajoutés, montrent que nombre des principaux auteurs chrétiens ont traité avant l'an mil, des relations avec les Juifs. On aura noté toutefois que la plupart de ces écrits proviennent du bassin méditerranéen. Et ils ne semblent pas avoir d'évidentes répercussions sur l'iconographie.

Au début du XI ${ }^{\mathrm{e}}$ siècle Fulbert de Chartres écrit “ contre les Juifs $\star^{6}$ et vers le troisième quart du siècle saint Pierre Damien, à

3. - La réappropriation du prophétisme par les imagiers chrétiens du XII* siècle ", Je monde de la Bible 131, novembre-décembre 2000, p. 47-59.

4. F. Vernet, - Juifs (controverses avec: les) „, Dictionnaire de théologie catholique 8-2, 1925, col. 1870-1917.

5. Altercatio Aecclesię contra Synagogam. Texte inédit du x" siècle ", B. Blumenkrany. éd., Revue du Moyen Âge latin 10, 1954, p. 4-159.

6. Patrologie latine, 1. 141, c. 307 et suiv.; B. Blumenkrany, A propos du (ou des) Tractatus contra Judaeos de Fulbert de Chartres ^, Revue du Moyen Age latin 8-1, 1952, p. 51-5/4. 
la requête d'un correspondant qui lui a demandé de réfuter les thèses juives, écrit un Antilogus contra Judaeos ${ }^{7}$. Un auteur anonyme a laissé un Dialogus inter Judaeum requirentem et Christianum e contrario respondentem ${ }^{8}$, et Samuel dit le Marocain, Juif converti à Tolède en 1085, écrit un De adventu Messiae quem Judaei temere exspectant liber ${ }^{9}$. Mais s'il n'y a pas de solution de continuité dans la production d'écrits relatifs à la question juive depuis les débuts de l'Église chrétienne, il n'en reste pas moins que les "disputes" entre Juifs et chrétiens ont été particulièrement nombreuses entre la fin du XI" siècle et le premier tiers du XIII" siècle. Au départ de cette période il y a eu le lancement de la première croisade par le pape Urbain II au concile de Clermont de 1095 et la Disputatio judaei cum christiano de fide christiana composée vers cette date, et sans doute en Angleterre, par Gilbert Crispin, abbé de Westminster, et par lui dédiée à saint Anselme, évêque de Cantorbéry ${ }^{10}$, importante par la trasmission objective des arguments juifs et par le fait qu'elle a été par la suite connue et copiée notamment par le pseudo-Guillaume de Champeaux, Jacob ben Reuben et Alain de Lille'1. A l'autre bout il y a l'interdiction faite par le pape Grégoire IX en 1233 aux simples fidèles de participer à des discussions religieuses avec les Juifs, la restriction aux " très bons clercs » par le roi saint Louis de la possibilité de " disputer » avec les Juifs (Joinville), le débat public ordonné en 1240 par le même roi Louis IX entre un groupe de chrétiens, conduit par Eudes de Châteauroux et le converti Nicolas Donin de La Rochelle, et un groupe de savants juifs mené par Rabbi Yehiel de Paris ${ }^{12}$. Il n'y a plus, en fait, dès lors de véritable débat, la cause étant d'avance entendue. Il en sera de même lors de la " dispute " de Barcelone en 1263. A la fin du siècle les Juifs seront expulsés d'Angleterre et de France. "Le XI" siècle avait été celui du dialogue relativement serein entre chrétiens et juifs - le texte le plus significatif étant celui de Gilbert Crispin. Le XII" siècle prolongeait ce dialogue mais le ton se faisait plus incisif... au dialogue succède la dis-

7. Patrologie Katine, 1. 145, c. 11-58.

8. Ibid. 1. 145, c. 57-68.

9. Ibid. 1. 149, c. 333 el suiv.

10. Ibid. . 1. 159, (. 1103-1112 : The Works of Gilbert Crispin abbot of Westminster, A. Sapir Abulafia el (i.-R. Evans éd. (Auctores britannici Medii Aevi, VIII). Oxford, 1986; B. Blumenkranz. * La Dispufatio Judei cum christiano de (jilbert Cirispin, abbe de Westminster s. Revue du . Moyen Âge latin IV. 1948, p. 237-252.

11. D. Berger. a Gilbert Cirispin, Nan of Lille and Jacob ben Reuben : a Study in the Transmission of Medieval Polemic .. Speculum 49, 1974. p. 34-17.

12. B. Blumenkranz, op. cit. (n. 1 ; , p. 47 . 
pute. Le XIII" siècle va être celui où la confrontation devient procès. $x^{13}$

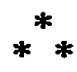

Mon attention sur l'influence des controverses judéochrétiennes sur l'iconographie a été attirée par l'étude que j'ai présentée à la Société des Fouilles archéologiques et des Monuments historiques de l'Yonne sur a la table d'or de la cathédrale de Sens "14. Cette superbe ceuvre d'art qui décorait le maître-autel de la cathédrale gothique (mi-XII's.) a été fondue en 1760 pour les besoins du roi lors de la guerre de Sept ans, mais un chanoine de la cathédrale en a fait alors une description minutieuse (fig. 1) et le chapitre a fait exécuter par le peintre Lambinet un dessin précis de l'œuvre (fig. 2). Or quatre des scènes de la table sont consacrées au martyre du patron de la cathédrale, Étienne, tel que nous le décrivent les Actes des Apôtres. Deux des scènes des angles supérieurs rapportent la « dispute » qu'Étienne eut avec les Juifs - avant sa lapidation, Juifs à gauche, Étienne à droite.

Disputare est un terme qui, dans la Bible, se trouve principalement dans les Actes des $\Lambda$ pôtres : pour les Juifs avec Étienne (Actes, VI, 9), pour Paul avec les Grecs à Jérusalem (IX, 29), pour Paul, à la Synagogue, à Athènes avec les Juifs (XVII, 17), à Corinthe avec les Juifs et les Grecs (XVIII, 4), à Éphèse avec les Juifs (XVIII, 19 et XIX, 8), à Troas lors d'une célébration de la fraction du pain (XX, 7,9), et encore à Césarée lors de l'emprisonnement de l'apôtre (XXIV, 12, 25) avant son transfert à Rome. Le titre des écrits se rapportant aux controverses judéochrétiennes de la fin du $\mathrm{XI}^{\mathrm{e}}$ et du $\mathrm{XII}^{\mathrm{e}}$ siècle est ordinairement disputatio : pour Gilbert Crispin, Odon de Cambrai ${ }^{15}$, Gilbert ${ }^{16}$, ou à Constantinople, Pascal Romain ${ }^{17}$, ou dialogus : pour Pierre

13. G. Dahan, introduction à l'édition-traduclion du /ivre des deux guerres du Seigneur et deux homélies de Guillaume de Bourges, Juif converti, écrit vers 1235 (Sources chrétiennes, n“288), Paris, 1981, p. 39 sq.

14. Bulletin de la Société des Fouilles archéologiques et des Monuments historiques de l'Yonne 18, 2001, p. 1-12.

15. Disputatio contra .Iudaeum, I.eonem nomine, de adventu Christi filii Dei, par Odon, évêque de (Cambrai (†1113), Patrologie latine, t. 160, c. 1103-1112.

16. Disputatio ecclesiae et synagogua, auctore Gillieberto, F. Martène et li. Durand éd., dans Thesaurus novus anecdotorum, t. 5, Paris, c. 1497-1506.

17. - Paschalis Romanus, disputatio contra Judeos „, G. Dahan éd., dans Recherches augustiniennes, vol. 11, 1976, p. 161.213 (1158 ou 1163). 


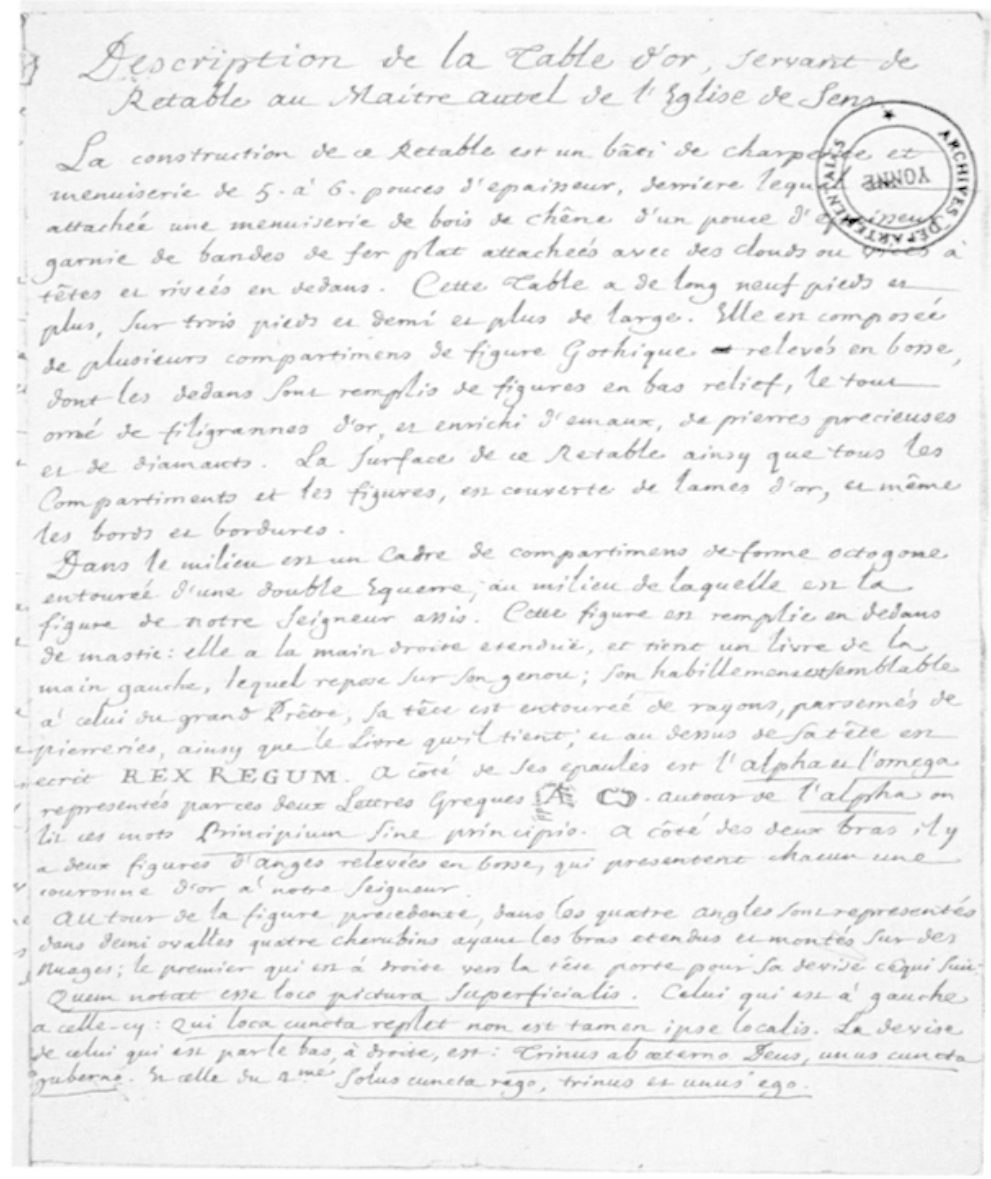

Fu: 1. - Description de la lable dor de Sins.

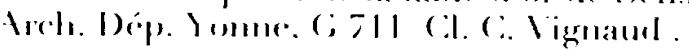

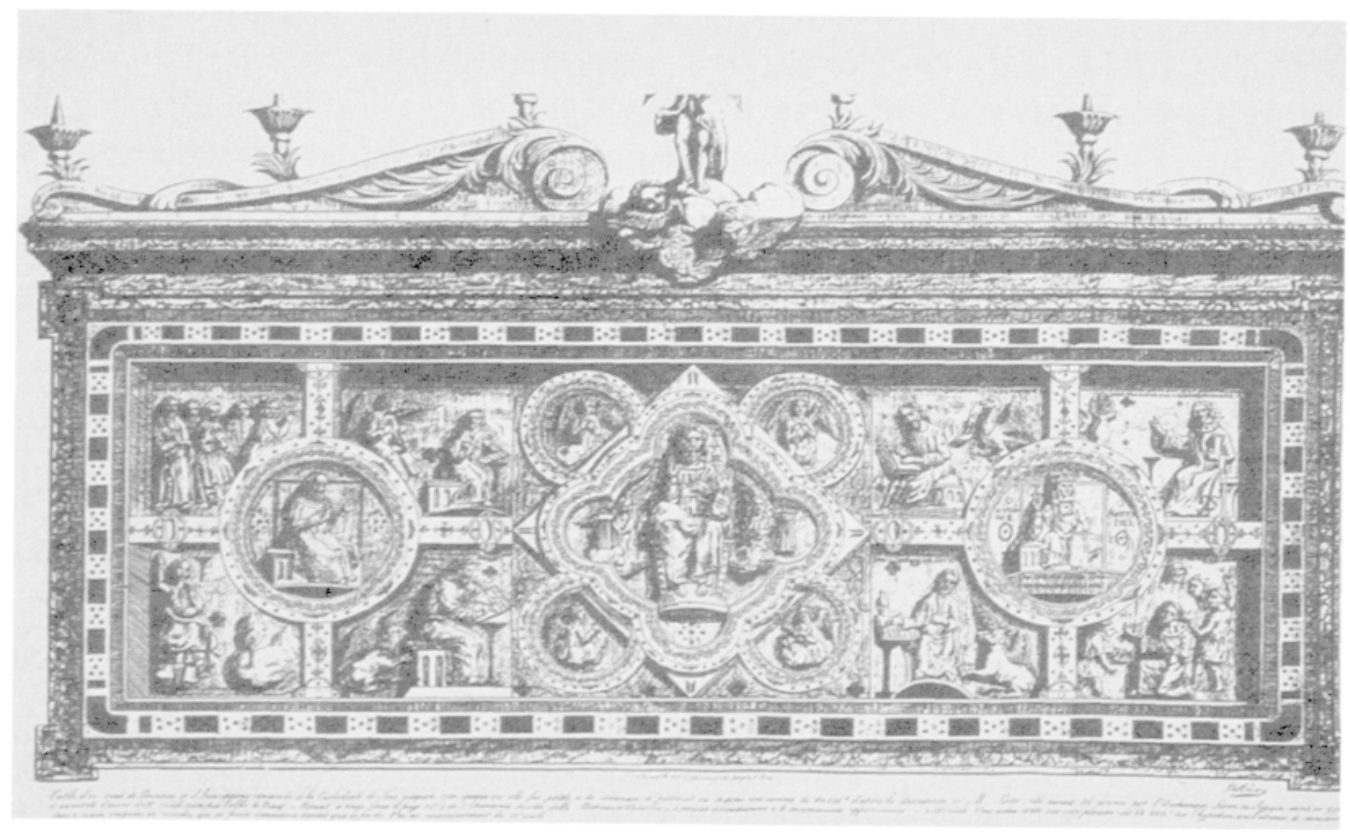

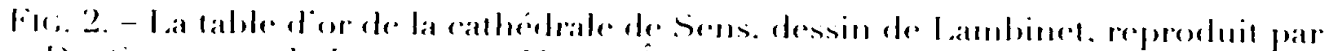

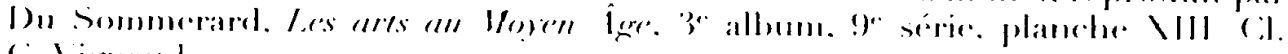
(:. I ignaturl. 
Alphonse ${ }^{18}$, le pseudo-Guillaume de Champeaux ${ }^{19}$, Rupert de Deutz $^{20}$, Pierre Abélard ${ }^{21}$, Gautier de Châtillon et Baudoin de Valenciennes $^{22}$.

Une plaque émaillée d'environ 1170-1180 au Victoria and Albert Museum à Londres présente Paul en discussion avec Grecs et Juifs : DISPUTABAT CUM GRECIS (Actes IX, 29), ET REVINCE. BAT JUDEOS (Actes XVIII, 28) ${ }^{23}$. La table d'or de Sens est plus précise. En, haut à gauche, « Les Juifs, tant scribes que pharisiens, docteurs de la Loi, disputent avec le soldat du roi suprême » :

\section{CERTANT JUDAEI TAM SCRIBAE QUAM PHARISAEI DOCTORES LEGIS CUM SUMMI MILITE REGIS.}

L'emploi du vers léonin a conduit à employer certare plutôt que le disputare des Actes des Apôtres ; ce qui est surtout significatif, c'est que les deux reproches formulés par les Juifs à l'encontre de la nouvelle religion ne figurent pas dans les Actes mais sont bien deux des reproches fondamentaux faits par les Juifs aux chrétiens :

NON VOLUIT VERE NASCI DEUS EX MULIERE

"Vraiment Dieu n'a pas voulu naître d'une femme ",

et

QUI CRUCE MORTUUS EST NON DEUS ESSE POTEST

«Celui qui est mort en croix ne peut être Dieu ».

Les Juifs, fidèles du Yaveh de l'Ancienne Alliance, ne pouvaient imaginer que Dieu eut pris, par la naissance dans le sein d'une femme, et qui plus est d'une vierge, la nature humaine. Dès les premiers siècles Tertullien, Évagre, Maxime de Turin, Ildefonse de Tolède font état de cette critique des Juifs. On la retrouve

18. Dialogus Petri cognomento Alphonsi, ex Judaeo christiani, dans Patrologie latine, t. 157, c. 537 et suiv.

19. Dialogus inter christianum et judaeum de fide catholica, dans Patrologie latine, t. 163, c. $1045 \cdot 1072$.

20. Annulus sive dialogus inter christianum et judaeum, dans Patrologie latine, t. 170, c. 559-610.

21. Dialogus inter philosophum, judaeum et christianum, dans Patrologie latine, t. 178, c. $1609-1682$.

22. Dans la Patrologie latine, 1. 209, c. 423-458. Outre la toujours précieuse synthèse de F. Vernet citée supra, n. A, on pourra voir l'étude de B. Blumenkranz, Juifs et chrétiens dans le monde occidental, 130-1096, Paris-La Haye, 1960, ou Judaism on Trial Jewish-Christian. Disputations in the Middle Ages, éd. et trad. H. Maccoby, Londres, 1993 (les \& disputes * de Paris 1240, Barcelone 1263, Tortosa 1413-1414). - I a controverse religieuse entre les chrétiens et les Juifs au Moyen Âge », avait déjà été présentée par I. Loeb dans la Revue d'Histoire des Religions 17, 1888, p. 311-337 ; 18, 1888, p. 133-156.

23. English romanesque art 1066-1200, Londres, 1984, n"290 et 290', p. 274. 
naturellement à la grande époque des controverses judéochrétiennes. Guibert de Nogent s'en fait l'écho dans son Tractatus de Incarnatione contra Judaeos ${ }^{24}$ : "Nul, disent les Juifs, sinon quelqu'un qui aurait perdu l'esprit, ne croit que Dieu ait voulu s'abandonner à l'insignifiance du ventre d'une femme et supporter les délais d'un développement ordinaire. Cela surtout est particulièrement horrifiant que celui qui est Dieu soit venu au monde par les parties sexuelles d'une femme... Si de plus il est dit né d'une vierge, c'est radicalement faux parce qu'un homme ne peut naître contre les lois de la nature. "Odon, évêque de Cambrai, fait dire à son Juif Léon : "En cela surtout nous nous moquons de vous et vous jugeons insensés. Car vous dites que Dieu a été enfermé dans un sein maternel, en l'indécente prison d'un ventre fétide, qu'il a souffert neuf mois et enfin que, le dixième mois, il en est sorti par une honteuse issue (qui ne peut être vue sans confusion), portant à Dieu une bien grande ignominie. ${ }^{2 \pi}$ Dans le Sefer ha-berit, "Livre de l'alliance ", Joseph Qimhi, qui a fui l'Espagne musulmane et s'est établi en 1148 à Narbonne, met en scène un interlocuteur juif qui se montre choqué de la croyance du chrétien en l'Incarnation : "Comment croirais-je que le Dieu grand, caché et voilé se soit établi sans nécessité dans le ventre d'une femme, dans des entrailles impures et immondes, que le Dieu vivant soit enfanté par une femme, enfant sans intelligence et sans discernement... Je ne crois pas en celte foi qui est tienne, car ma raison ne me donne pas le droit de diminuer la grandeur de Dieu - qu'll soit exalté ", et son contemporain Jacob ben Reuten affirme, lui aussi, dans son Sefer milhamot Adonay, "Livre des guerres de Dieu ", qu' « il est blâmable et blasphématoire de prétendre que Dieu ait pu être enfermé dans des entrailles et ainsi privé de lumière ${ }^{26}$.

Même incompréhension des Juifs vis-à-vis de la mort du Christ sur la croix. Tertullien et Évagre nous disent qu' i ils ne peuvent croire que Dieu ait exposé son fils à ce genre de mort ", selon ce que dit le Deutéronome (XXI, 23) : " Il est maudit de Dieu celui qui est pendu sur le bois ${ }^{27}$. Dans l'Altercation anonyme du

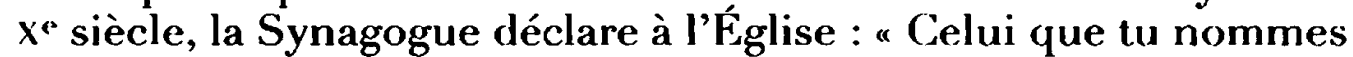
Christ, d'où tu as ton nom, que tu dis né d'une vierge, a souffert

24. Patrologie latime, 1. 156, c. 492 .

25. Op. cir. (n. $15 \mathrm{j}_{0}$ (. 1110.

26. (*. Dahan. Saint Anselme, les Juifs, le judaïsme *. dans Les mutations socio-

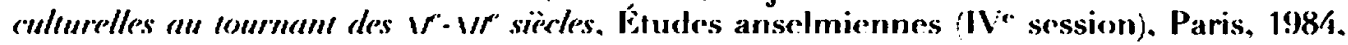
p. $527 \mathrm{sq}$.

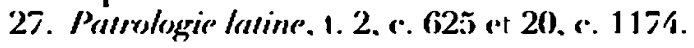




\section{COMPTES RENDUS DE L'ACADÉMIE DES INSCRIPTIONS}

opprobres et a été crucifié et livré à la mort. Comment pourrait-il me libérer et me sauver, lui qui n'a pu éloigner de lui tant d'opprobres et une telle mort ${ }^{28}$. Et le Juif converti Pierre Alfonse dira de même : "Comment a t-il pu souffrir d'être crucifié, et ne s'est-il pas arraché des mains des Juifs? Sa toute-puissance n'en est-elle pas ruinée. ${ }^{29}$

Dans l'angle supérieur de droite de la table d'or de la cathédrale de Sens, Étienne répond aux scribes et aux pharisiens :

« Né d'une vierge, Dieu a été condamné à la croix Cette loi l'atteste qui t'est follement donnée » IN CRUCE DAMNATUS DEUS EST EX VIRGINE NATUS HAEC LEX TESTATUR QUAE TIBI STULTE DATUR,

et anspiré par Dieu le martyr conclut que la loi hébraïque prend fin lorsque cesse l'onction royale * INSPIRANTE DEO MARTYR CONCLUDIT HEBRAEO FINIS ADEST LEGIS DUM DEFICIT UNCTIO REGIS.

Dans les deux angles inférieurs de la table d'or de Sens est représentée la lapidation d'Étienne. A gauche Saul garde les vêtements des bourreaux (Actes, VII, 58) avec l'inscription :

HIJNC. HABUIT SAIUIUM JIIVENFM I.FX GRATIA PAIIIIM CHRISTUS EUM LAVIT STEPHANI QUEM LAPIDAVIT

a La loi a tenu ce jeune Saul, la grâce a tenu Paul ${ }^{30}$, le Christ l'a lavé, à la prière d'Étienne qu'il a lapidé n.

A droite est représenté la mort d'Étienne qui dit :

TORRENTES LAPIDES AMPLECTOR UT HOSTIA FIAM SED NE DEFICIAM SUFFICIT UNA FIDES

a J'embrasse avec joie ce torrent de pierres qui fait de moi une hostie (victime), mais la foi seule suffit à m'empêcher de défaillir .

La mort d'Étienne est à l'image de celle du Christ : il pardonne à ses bourreaux (Actes VII, 60), comme le Christ l'avait fait sur la croix (Lc XXIII, 34), il remet son esprit au Seigneur (Actes VII, 59) comme le Christ entre les mains du Père (Lc, XXIII, 46), il est tué par les Juifs. Le pardon et l'appel au Seigneur sont repris dans le

28. Dans Revue du Moyen Âge latin 10, 1954, p. 87 sq. (supra, n. 5).

29. Patrologie latine, t. 157 , c. 639.

30. C.f. Jn, I, 17. 
tympan de l'église Saint-Étienne de Mehringen consacré à la lapidation d'Étienne (vers 1130-1140) : LAPIDABANT JUDEI STEPHANIUM INVOCANTEM ET DICENTEM DOMINE JHESU SUSCIPE SPIRITLM MEL;M ${ }^{31}$.

Une ceuvre d'ivoire de la seconde moitié du XII" siècle, la croix de Bury St Edmund ${ }^{32}$, fait expressément état de l'incrédulité des Juifs devant la mort de Celui que les Chrétiens présentent comme Fils de Dieu :

\section{+ JUDEI RISERE DEI PENAM MORTIS}

"Les Juifs se gaussèrent de ce que Dieu eut subi la peine de mort "

tandis qu'une autre inscription reprend le texte du Deutéronome (XXI, 23) :

\section{MALEDICTIS OMNIS QUI PENDET IN LICNO}

"Maudit tout homme qui pend sur le bois " 33 ,

qui est celui-là même que les Juifs opposaient à Tertullien, Évagre, saint Justin pour nier la possibilité de la mort du Fils de Dieu. Une autre ceuvre des environs de 1160, l'autel portatif de Stavelot, rappelle la culpabilité des Juifs, qui fut, disaient les auteurs chrétiens, cause de leur dispersion à travers le monde : en bas de la plaque d'autel consacrée à la Passion Jésus est figuré devant Pilate qui dit : INNOCENS EGO SUM $\Lambda$ SANGUINE (Mt XXVII, 24), a Je suis innocent de ce sang c'est votre affaire ", tandis que tout le peuple juif répond: SANGUIS EJUS SUPER NOS ET SUPER FILIOS NOSTROS (Mt XXVII, 25), " Nous prenons son sang sur nous et sur nos enfants ${ }^{34}$. Le rapport avec les controverses judéo-chrétiennes est ici confirmé par l'aveuglement de la Synagogue. A la cathédrale de Châlons-sur-Marne un vitrail de la

31. Ii. Neubauer, Die romanischen skulptierten Bogenfelder im Sachsen und Thüringen, Berlin, 1972, p. 16is et fig. 117.

32. I a eroix anglaise de Bury St. Edmond a été acequise en 1963 par le Metropolitan Museum of Art de New York. Voir W. Mersmann, a Das Elfenbeinkreuz der Sammlung Topic-Mimara „, Wallref-Richartz Jahrbuch 25, 1963, p. 7-108, ill.; ot Th. P. F. Iloving, - The Bury St Edinunds (Cross „, The Metropolitan Museum of Art Bulletin, 19614. p. 317.340, ill.

33. I a citation exacte de la Vulgate est : Maledictus a Deo est qui pendet in ligmo. Fille est reprise par Paul (Galates, III, 13;.

34. Art mossen aur or ot vir sièrles, par S. Collon-Gevaert, J. I cejeune et J. Stiennon, Bruxelles. 1971. p. 208 : : M.-M. Gauthier, Emaux du . Koyen Âge occidental, 2 " ćl., Fribourg, 1972. n" 95. p. 351 sq. Cf. Maxime de "Turin. Contra Judaesos, dans Patrologie latine, t. 57, c. 800 : Sanguis ejus super nos ef super filios nostros, wobis ad condemnationem, nobis vero ad salutem : I. Grodecki, * I,es vitraux de la cathédrale de Châlons-sur-Marne: s, Bulletin de la Sinciété nationale des Antiquaires de France. 1950-1951, p. 196-203. 
Crucifixion du milieu du XIl" siècle comporte aussi une figure de la Synagogue qui dit de même :

SANGUIS EJUS SUPER NOS ET SUPER FILIOS NOSTROS ${ }^{35}$.

La faute des Juifs, c'est aussi celle de la trahison de Judas que rappelle un chapiteau roman de l'abbatiale de Sorde-l'Abbaye :

\section{EU ! JUDAS [I]SCHARIOT \\ + DEI TRADICCIO DOMINI NOSTRI JHESU CHRISTI ${ }^{36}$,}

"Malheur! c'est Dieu lui-même qu'il livre en la personne du Christ ». A la façade de Santa Maria la Real à Sangüesa, Judas est représenté pendu, avec sur sa poitrine les mots JUDAS MERCATOR, "Judas le marchand ", ce dernier texte accompagnant également la Synagogue sur un vitrail de la cathédrale de Châlonssur-Marne ${ }^{37}$. Il faut rappeler ici ce que dit le Décret de Gratien, qui est contemporain des sculptures de Sangüesa : « En chassant du Temple ceux qui y vendaient et achetaient, le Seigneur a signifié qu'un homme marchand ne peut qu'avec peine ou jamais plaire à Dieu. Aucun chrétien donc ne doit être marchand, ou, s'il a voulu l'être, qu'il soit chassé de l'Église de Dieu. "38 Jusqu'au concile de Vatican II on lisait à l'office des ténèbres du jeudi Saint : le répons : Judas mercator pessimus osculo petiit Dominum, « Judas le marchand sacrilège s'approcha de Jésus pour le baiser ».

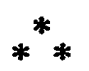

La représentation du martyre d'Étienne sur la table d'or de la cathédrale de Sens ne met pas seulement en cause l'incrédulité des Juifs face à l'Incarnation et à la Passion. Ce double refus c'est évidemment aussi le refus de croire en la venue du Messie, et c'est encore l'attachement répété à la seule loi de Moïse. L'affirmation d'Étienne sous “ l'inspiration de Dieu » est claire sur ces deux points : " la loi hébraïque prend fin lorsque cesse l'onction royale."

35. I. Grodecki, Le vitrail roman, Fribourg-Paris, 1977, p. 278.

36. Corpus de la France médiévale, 6, R. Favreau, B. I.eplant et J. Michaud éd., Paris, 1981 , n"15, p. 105.

37. Statue-colonne la plus à droite du portail sud de Santa Maria la Real, inscription sur la poitrine de Judas (Poitiers, Centre d'Études supérieures de Civilisation médiévale, photothèque).

38. Decretum magistri Gratiani, E. L. Richter et E. Friedberg éd., Leeipzig, 1922, c. 308 (prima pars, dist. 88, cap. 11). 
Cette affirmation de la fin de l'onction royale introduit directement à un élément essentiel du débat judéo-chrétien. Elle renvoie au chapitre IX de Daniel où l'ange Gabriel enseigne au prophète qu' “ il a été fixé soixante-dix semaines sur son peuple et sur sa ville sainte, pour mettre un terme au péché... et oindre un saint des saints " - et ungatur Sanctus sanctorum. " Depuis la sortie de la parole de rétablir et de bâtir Jérusalem jusqu'à un Christ chef, il y a sept semaines... et après soixante-deux semaines le Christ sera tué sans qu'il y ait eu de faute. Et le peuple d'un chef qui viendra détruira la ville et le sanctuaire. »On comprend généralement les semaines annoncées comme des semaines d'années, c'est-à-dire que soixante-dix semaines correspondent à $70 \times 7$, soit 490 ans, et on doit se rappeler que le mot Messie veut dire "oint ", en grec "Christ. ". L'interprétation messianique de ce texte hermétique", telle qu'elle a cours au Moyen Âge, voit dans les sept semaines (49 ans), le temps de l'exil à Babylone (586-536 av. n. è.), dans les soixante-dix semaines ( 490 ans), le temps entre le décret d'Artaxerxès Ier de $\mathbf{4 5 8}$ permettant la reconstruction des murs de Jérusalem et la mort du Christ en 32 de notre ère, la dernière annonce étant celle de la destruction de Jérusalem par Titus en 70 . Aussi cette prophétie de Daniel sera continuellement opposée aux Juifs qui soutiennent que le Messie n'est pas encore venu. On la trouve citée chez Tertullien, Évagre, Quodvultdeus, Isidore de Séville, dans l'Altercation de l'Église contre la Synagogue (X's.), chez Fulbert de Chartres, Pierre Damien, Samuel dit Marochianus, Guibert de Nogent, Pierre Alfonse, le pseudo-Guillaume de Champeaux, Gautier de Châtillon, Pierre le Vénérable ${ }^{40}$, Alain de Lille, l'anonyme Traité contre les Juifs, Guillaume de Bourges, Matfré Ermengaud $^{11}$. Mais naturellement cette interprétation chrétienne de la prophétie de Daniel sera toujours rejetée par les auteurs juifs.

On aura noté que Daniel parle de l'onction du Saint des saints, alors que l'inscription de la table d'or de Sens évoque la cessation

39. E. Philippe, " Daniel ", dans Dictionnaire de la Bible, 1. 2, F. Vigouroux dir., Paris. 1910, e. 1277-1282 ; A. I acoeque, Le livre de Daniel (Commentaire de l'Ancien T'estament, $X V b$, Paris, 1976, p. 130-1:20 ; B. Blumenkran\%, op. cit. (n. 22;, p. 245 sqq. Le versel 26 du chapitre 9 de l)aniel est inserit dans les peinlures murales de Sant'Angelo in Formis, et sur la croix de: Bury St Edmund. ainsi que: le retable de: Klosterneuburg.

40. Pierre le Vénérable, Tractatus adsersus Judaeorum inveteratam duritiem, dans Patrologie latime 1. 189. c 558, 563-565: citant Daniel (chap. IX), comme Tertullien l'avait déjà noté, il écrit : Ecre nom solum annum adventus (hristi, set et passionis ar mortis ejus tempus vester prophera describit.

41. B. Blumenkran\% * Feriture et image dans la polémique antijuive de Matfré lirmengaud n, dans Juifs et judeüsme du languedor (Cahiers de Fanjeaux, 12, Toulouse, 1977 p. 30 ). 
de l'onction. Il faut ici se reporter à un texte fondamental pour le sujet, le "Sermon contre les Juifs, les païens et les ariens ", longtemps attribué à saint Augustin, mais aujourd'hui restitué à son contemporain, l'évêque de Carthage Quodvultdeus ${ }^{42}$. Pour convaincre les Juifs de la venue du Messie, celui-ci fait intervenir successivement une série de témoins de l'Ancien Testament : ‘ $O$ Juifs, jusqu'à aujourd'hui vous niez le Fils de Dieu... Cherchez témoignage sur le Christ. Dans votre Loi il est écrit que le témoignage de deux hommes est véridique ${ }^{43}$. Que procèdent de votre Loi non seulement deux, mais davantage de témoins du Christ, et qu'ils convainquent les auditeurs de la Loi. » Lorsque Daniel est appelé il dit: “Lorsque viendra le Saint des saints, l'onction cessera „, cum venerit Sanctus sanctorum cessabit unctio, c'est-à-dire qu'à partir de la venue du Christ il n'y aura plus de rois de Judas qui auraient reçu l'onction. Ce retournement du sens de la prophétie de Daniel est parfaitement illustré par une miniature du manuscrit latin 10433 à la Bibliothèque nationale de France, où le registre supérieur est occupé par la scène de l'onction de David tandis qu'au registre inférieur un personnage pose, sur un phylactère la question même du Sermon de Quodvultdeus :

DIC, SANCTE DANIEL, DIC DE CHRISTO QUOD NOSTI TESTIMONIUM VERITATIS

" Dis, saint Daniel, dis ton témoignage de vérité sur le Christ ",

à quoi Daniel répond :

CUM VENERIT SANCTUS SANCTORUM CESSABIT UNCTIO ${ }^{44}$ (fig. 3).

Quand, dans une inscription, dans un ouvrage-c'est le cas pour les ouvrages contre les Juifs de Pierre Damien, de Gilbert Crispin et du pseudo-Guillaume de Champeaux - on trouve la prophétie de Daniel citée sous la forme de "cessation de l'onction "on peut tenir pour assuré que la source en est le « Sermon contre les Juifs " de Quodvultdeus. Au cours du dernier quart du XIX" siècle Marius Sepet a montré que le théâtre du Moyen Âge avait son origine

12. Opera Quodvutdeo Carthaginiensi episcopo tributa, R. Braun éd. (Corpus christianorum, Series latina, I.X), Turnhout, 1976, p. 225-258; Patrologie latine, 1. 12 (ceuvres de saint Augustin), (.. 1123-1128.

43. Deutéronome XVII, 6 et XIX,15.

14. (. M. Kaufimann, Romanesque Manuscripts 1066-1190, Londres, 1975, p. 114 sq., ill. 255 (A Survey of Manuscripts Illuminated in the British Isles, III). Voir aussi F. Gay, Les prophètes. Épigraphie, doctorat de $3^{*}$ cycle, dact., Poitiers, 1980. 


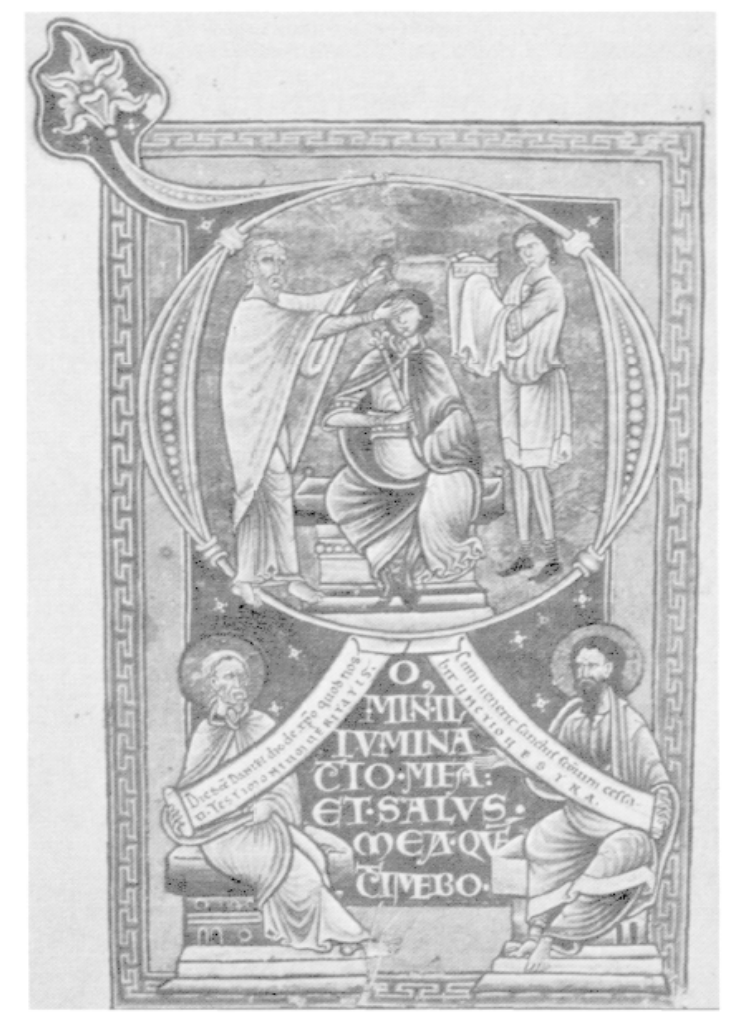

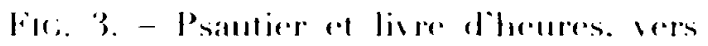
1170. dapres (:. M. Kauffimamo. Romme-

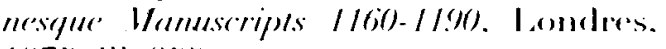
1975. ill. 255.

dans les representations dramaliques organisés d'abord aulour

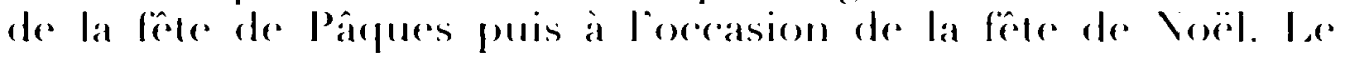

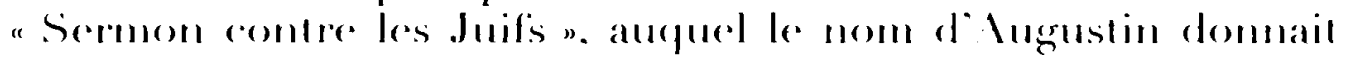

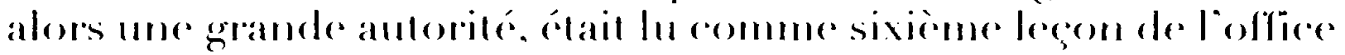

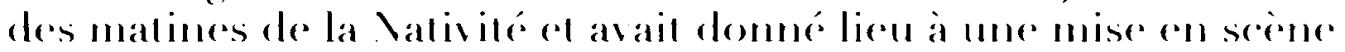

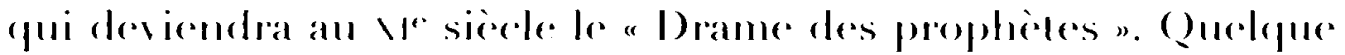
lomps apres Julien I Iuand allimail latlention sur le lait que les lextes des prophedes du "Semon comtere les Juits ". repris dans le "Drance des propheles " areompagnadent une serie de progrant-

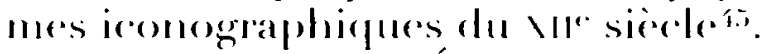

Irthur K. Porter. Emile Vâle. Karl Yomegiri on popularise les chudes de Sépel el Dumand. la "Drame des prophèles" apparaîl

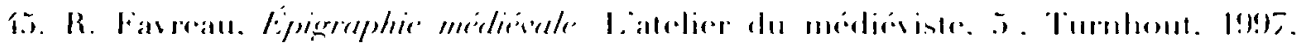
p. $22.5-230$.

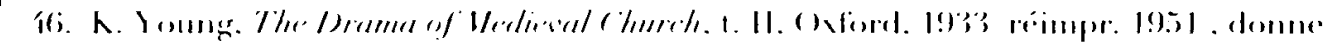
les lendes de a.es premiers "mistiones" 
dans la seconde moitié du XI $I^{e}$ siècle à Saint-Martial de Limoges : le préchantre de la célèbre abbaye appelle les prophètes à comparaître devant les Gentils et les Juifs, et chacun s'avance et chante son témoignage, celui-là même du “ Sermon » de Quodvultdeus ${ }^{47}$. Il sera joué, aux XII ${ }^{e}$ et XIII ${ }^{e}$ siècles dans les cathédrales de Salerne, de I Laon, de Rouen. Un autre drame liturgique, le Sponsus ou “Drame de l'époux » ou « Mystère des vierges sages et des vierges folles ", remonte lui aussi au XI ${ }^{e}$ siècle, et son unique manuscrit, qui comporte une précieuse notation musicale, provient également de Saint-Martial de Limoges. Il met en scène les vierges sages et les vierges folles; quand l'époux arrive les vierges folles sont précipitées dans l'enfer et alors interviennent successivement tous les personnages du a Sermon » de Quodvultdeus avec les citations mêmes du Sermon ${ }^{48}$. Le a Mystère d'Adam ", daté selon ses éditeurs de 1225-1250 ou de 1125-117549, n'est autre que la mise en scène de l'histoire d'Adam et d'Ève et d'Abel et de Caïn, suivie du "Drame des prophètes ». A la fin du Jeu de Daniel (Beauvais, vers 1140) le prophète reprend son annonce de la fin de l'onction. La grande vogue du drame liturgique se situe aux $\mathrm{XII}^{\mathrm{e}}$-XIII' siècles $^{50}$. C'est aussi la grande période des controverses judéo-chrétiennes, et le a Sermon " de Quodvultdeus est un sermon contre les Juifs. La question mérite donc d'être posée : les controverses judéo-chrétiennes n'ont-elles pas eu un rôle dans l'apparition du « Drame des prophètes » et dans sa représentation sur une série de façades ou autres œuvres d'art des XII'-XIII siècles ? Or on ne la trouve pas posée, et Émile Mâle se borne à écrire que les prophètes sculptés à la façade s'adressent au passant, au Juif, à l'incrédule ${ }^{51}$.

Les témoins présentés aux Juifs par le « Sermon » de Quodvultdeus se succèdent avec des textes qui diffèrent plus ou moins de la traduction latine qu'établit alors saint Jérôme, traduction qui ne

47. Le drame de la Passion apparaît aussi au $\mathrm{xI}^{\circ}$ siècle, en l'abbaye du Mont-Cassin (S. Sticca, The J atin Passion Play. Its Origin and Development, Albany, 1970).

18. Edité par I. J. N. Monmerqué et F. Michel, Théâtre français du Moyen Age, Paris, 1839, p. 3-9, et par W. Cloetta, Le Mystère de l'F́poux „, Romania 22, 1893 , p. 177. 229.

19. Le mystère d'Adam (ordo representacionis Ade), P. Aebischer éd. (Textes littéraires français, 99), Genève-Paris, 1963, avec la datation du xu" siècle; (C. J. Odenkirchen, The Play of Adam. Ordo representacionis Ade, Brookline (Mass.)-I eyde, 1976, avec la datation du xur" siècle.

50. K. Young, op. cit. (n. 46), II, p. 301 (Jeu de Daniel) ; C. Verzar Bornstein, Portats and Politics in the early Italian City-State: The Sculpture of Nicholaus in Context, Parme, s.d., p. 100.

51. L'art religieux au XIr' siècle en France. Étude sur les origines de l'iconographie du Moyen Äge, 2* éd., Paris, 1924, p. 145. 
s'imposera de façon générale (la Vulgate) que plusieurs siècles plus tard :

- Isaie : Ecce virgo in utero concipiet et pariet filium, et vocabitur nomen ejus Emmanuel Quod est interpretatum Nobiscum Deus (VII, 14). Vulgate : Ecce virgo concipiet.

- Jérémie : Hic est Deus noster et non aestimabitur alius absque illo, qui invenit omnem viam scientiae, et dedit eam Jacob puero suo et Israel dilecto suo. Post haec in terris visus est, et cum hominibus conversatus est (Baruch, III, 36-38). Vulgate : alius adversus eum. Hic adinvenit... et tradidit illam Jacob...

- Daniel : Cum venerit Sanctus sanctorum cessabit unctio. Vulgate : Et ungatur Sanctus sanctorum (IX, 24).

- Moïse : Prophetam vobis suscitabit Deus de fratribus vestris. Omnis anima quae non audierit prophetam illum exterminabitur de populo suo. Vulgate: Prophetam de gente tua et de fratribus tuis sicut me suscilabit tibi Dominus Deus tuus (Deutéronome, XVIII, 15). Exterminabitur de populo suo ef. (Exode XXX, 33).

David : Adorabunt eum omnes reges terrae; omnes gentes servient illi (Ps LXXII [LXXI], 11 ; Vulgate : ei)

- Habacuc: Domine, audivi auditum tuum et timui ; consideravi opera tua, Deus, et expavi. Vulgate: Domine, audivi auditionem tuam et timui (III, 2).

Après les témoins “ de la Loi " le “Sermon" présente aux Juifs des témoins “ de leur race " (ex gente vestra), Siméon, Zacharie et Élisabeth, Jean Baptiste, et des témoins " d'une autre race ou nation ", Virgile - Jam nova progenies coelo demittitur alto (Eglogue, 4, v. 7) -, Nabuchodonosor, les Sibylles - Judicii signum, etc.

Ces derniers témoins apparaissent peu dans l'iconographie du XII" siècle. On notera cependant qu'à la façade occidentale de la cathédrale de Laon on a et le vers ci-dessus de Virgile, et le 2r vers des oracles sibyllins (...PER SAECLA FUTURUS). Comme il y a aussi Nabuchodonosor et les trois jeunes Hébreux dans la fournaise (TRES PUERI IN FORNACE), Habacuc, des prophètes, et que l'on conserve dans un manuscrit liturgique de Laon un « Drame des prophètes » avec les personnages et citations du "Sermon » de Quodvultdeus ${ }^{52}$, on peut penser à une influence directe du "Sermon " sur cette iconographie. Mais je voudrais surtout insister sur une série d'cuvres, pour la plupart du XII' siècle, où figurent les prophètes du "Sermon " et du "Drame des prophètes " avec les textes mêmes de Quodvultdeus.

52. Ordinuires de l'église cathédrale de laon (1ur et vur' s.j suivis de deur mystères liturgiques. I'. Chevalier éd. 'Bitslionhèque liturgique, 6 , Paris. 189\%, p. 385-389. 
Sur les côtés de l'autel portatif d'Eilbert de Cologne au Kunstgewerbemuseum de Berlin, vers 1150 , sont représentés dix-sept prophètes et grands personnages de l'Ancien Testament, avec des textes pour seize d'entre eux. Une inscription avertit que ces personnages de l'Ancien Testament, a inspirés par le ciel pour qu'ils prophétisent au sujet du Christ, ont prédit ce qui devait arriver ». Les textes qui accompagnent Isaie (VII, 14), Jérémie (Baruch, III, 38), et Daniel (d'après IX, 24 : CUM VENERIT SANCTUS SANCTORUM CESSABIT UNCTIO) sont ceux-là mêmes que citent Quodvultdeus.

Aux jambages du portail principal de la cathédrale de Crémone $^{\mathfrak{5}^{3}}$ sont figurés à gauche Isaïe et Jérémie, à droite Ézéchiel et Daniel. Le texte d'Isaie (VII, 14) est celui que donne Quodvultdeus, mais c'est un texte constamment cité à propos de la maternité de Marie. L'emprunt à l'évêque de Carthage est évident pour les textes de Jérémie (Baruch, III, 36-37) et de Daniel (d'après IX, 24). En effet la citation de Jérémie comprend un inquid, qui est dans le sermon et ne peut s'expliquer sans cette référence : "Celui-ci, dit-il, est notre Dieu... » Quant à Daniel on a gravé la question même du Sermon de Quodvultdeus : « Dis, saint Daniel, ce que tu as connu du Christ n et la réponse se présente sous la forme même du Sermon: "Lorsque viendra, dit-il (inquid), le Saint des saints, votre onction cessera. „ On notera que le unctio vestra ne peut se comprendre autrement que comme une adresse aux Juifs. Ézéchiel ne figure pas dans le Sermon, mais le texte qu'il cite $^{54}$ se trouve dans de nombreux dialogues ou disputes entre Juifs et chrétiens. Les mêmes quatre grands prophètes se retrouvent, au portail occidental de la cathédrale de Ferrare, avec les mêmes textes, les mêmes renvois évidents au Sermon et aux Juifs - ECCE, INQUID, DEUS...; DIC, SANCTE DANIEL, DE CRISTO ; UNCCIO VESSTRA ${ }^{55}$. On notera qu'à Ferrare on a aussi Gabriel et Marie, donc également référence à l'Incarnation, un point que les Juifs récusent absolument. Au portail ouest de la cathédrale de Vérone, à gauche (fig. 4), les textes cités par Isaïe, Jérémie et Daniel sont ceux du Sermon de Quodvultdeus, avec, là aussi, le rappel évident de l'emprunt pour Jérémie: ECCE, INQUID, DEUS NOSTER..., « Voici, dit-il, Notre Dieu... » Figure encore au portail Habacuc, avec la citation caractéristique à partir

53. A. K. Porter, Lombard Architecture, t. II, New Haven-I,ondres-Oxford, 1916, p. 386 sq.

54. Fzéchiel, XIIV, 1-2. Ce texte se trouvait aussi à la cathédrale de Fidenza, la statue étant aujourd'hui au Musée Gazzola (A. K. Porter, op. cit. [n. 53], t. III, p. 251).

55. Id., op. cit. (n. 53), t. II, p. 419 sq. ; Ch. Verzar Bornstein, op. cit. (n. 50), p. 100. 


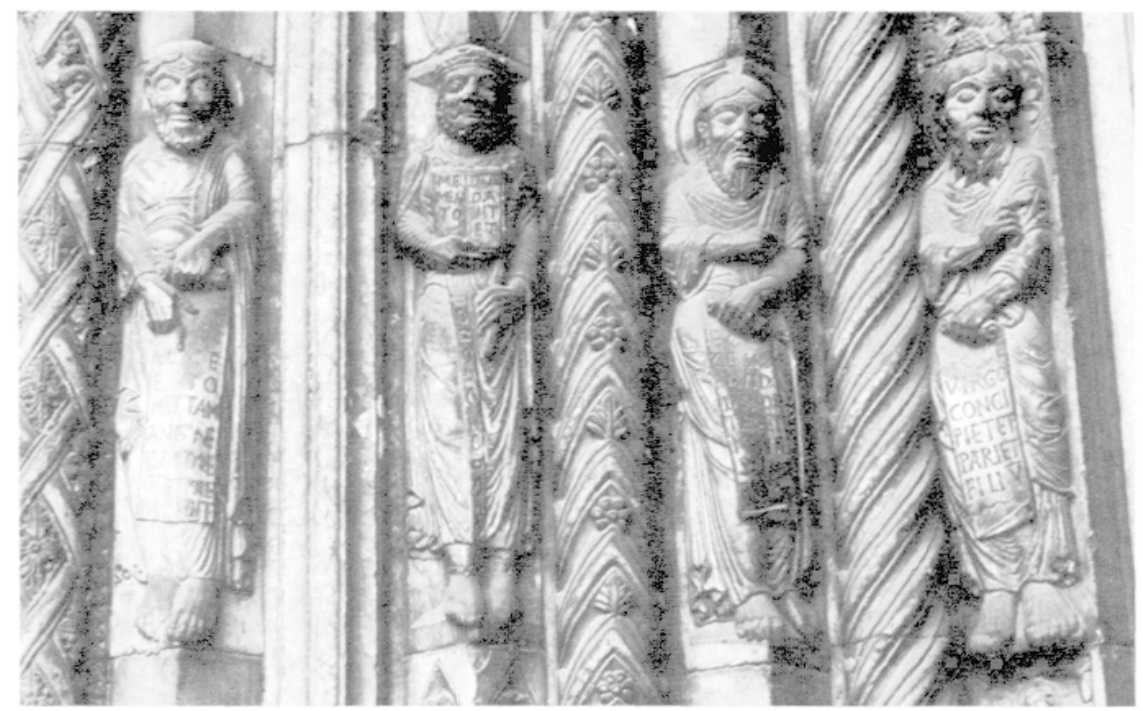

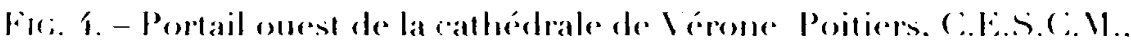
phostothiogure.

de (Quodeuddeusiti. Sur la chaire de San leonardo in Ireelri, a

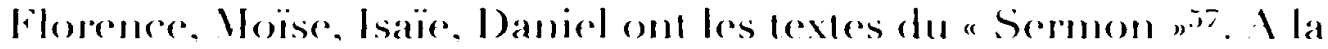
eoupole de lefommanued a San Vareo de lenise lsäre Jérémie el Daniel présentent les fextes de (2uodvultdeus, el il en est de même pour Isaie el Daniel sur la Pala d'orois. A la Martomana a Palemes. Jéremier. Isaïe ot llöse sont aceompagnés des cilations, en greer. du "Sermon" que lon retrouse pour Isäe el Jéremie - aver

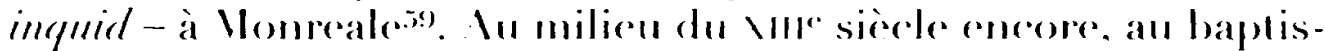

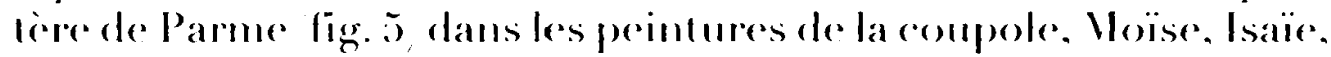

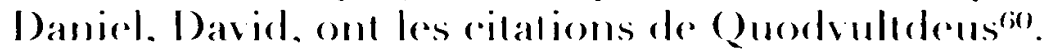

Si les exemples sont particulierement nombremx el significatifs

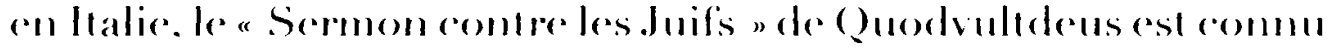

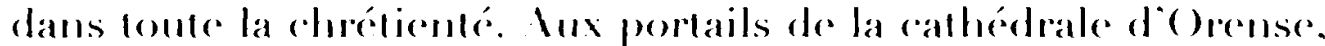
en Calice. (osée. Jomas, Jéremie soml seulement identilies par leur nom, Isaie ef I aniel le sont par les citations du "Sermon "iol

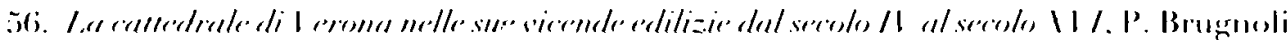
dir.. I enise. 1!187. p. 1 亿2 sq.

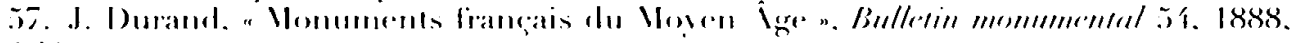

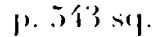

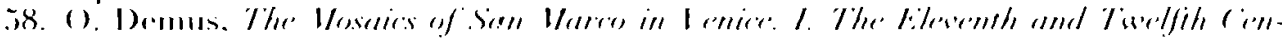

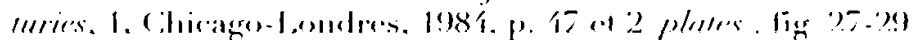

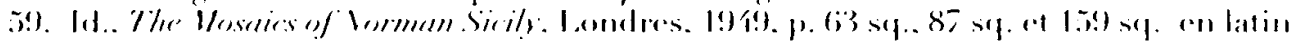
pour Vomerale.

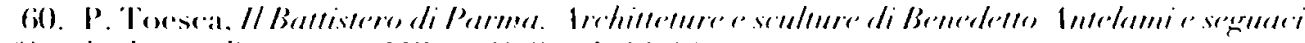

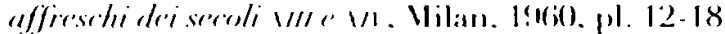

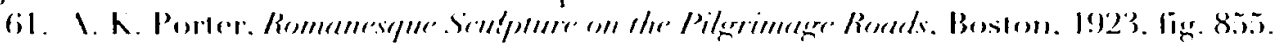




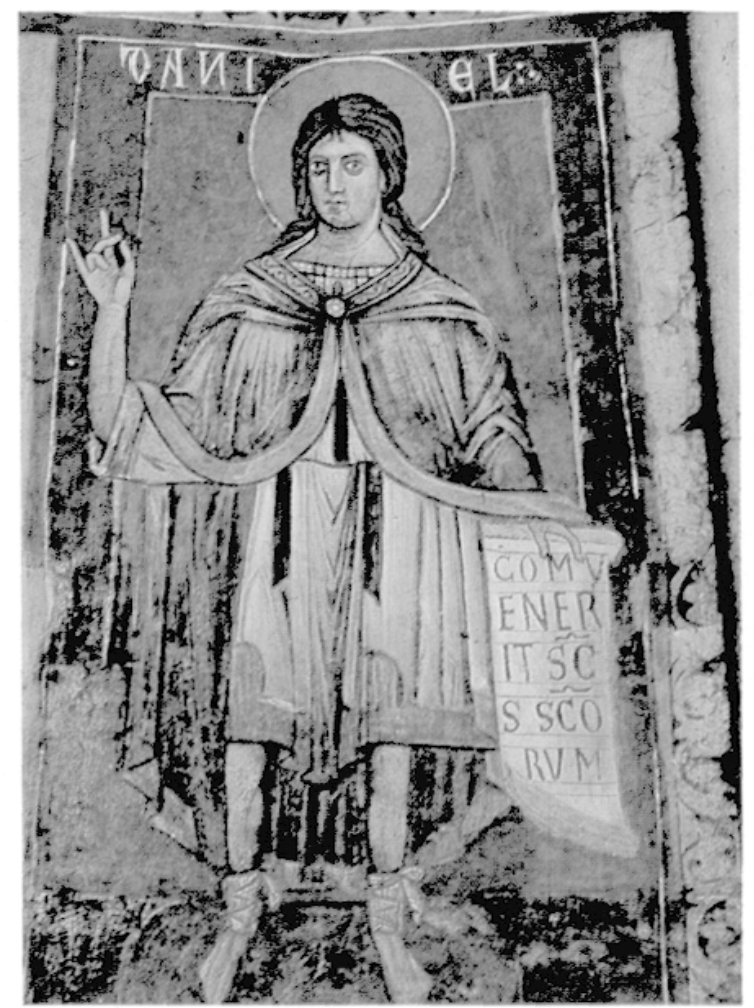

Fis. :. - Baptistere de Parme, le prophete Daniel, diapres P. Tonesca. // Batlistero di Prormme... Vlilan. 1960. p.1. 1\%.

fige 6, au grand portail de la cathédeale de Tarragone les cilations

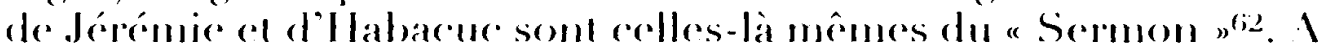
la frise de Votre-Dame-la-Cirande a Poitiers les lextes inserits sur

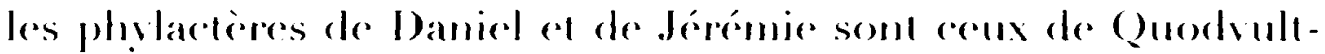

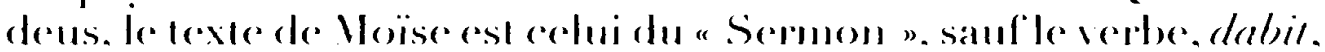

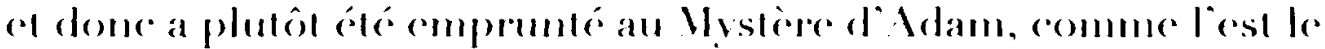
lexte d'lsaite. "un rameall sortima de la sourhe de Jesse " XI, I. sans doute retenu du fait de la présenere dans la suite de la frise, de

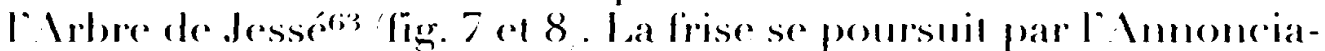

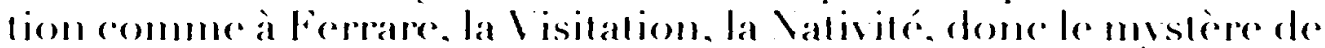
Inearnation que les Juilis estiment indigne dre la grandeur de Dicul.

las emprouls perment elre plus limilés. mais signilicalifis

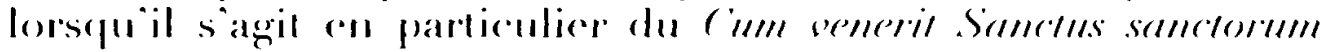

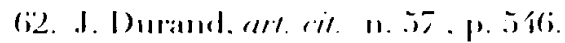

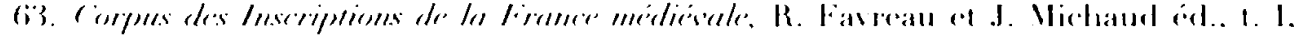

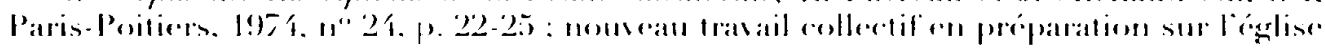
pollitall. 


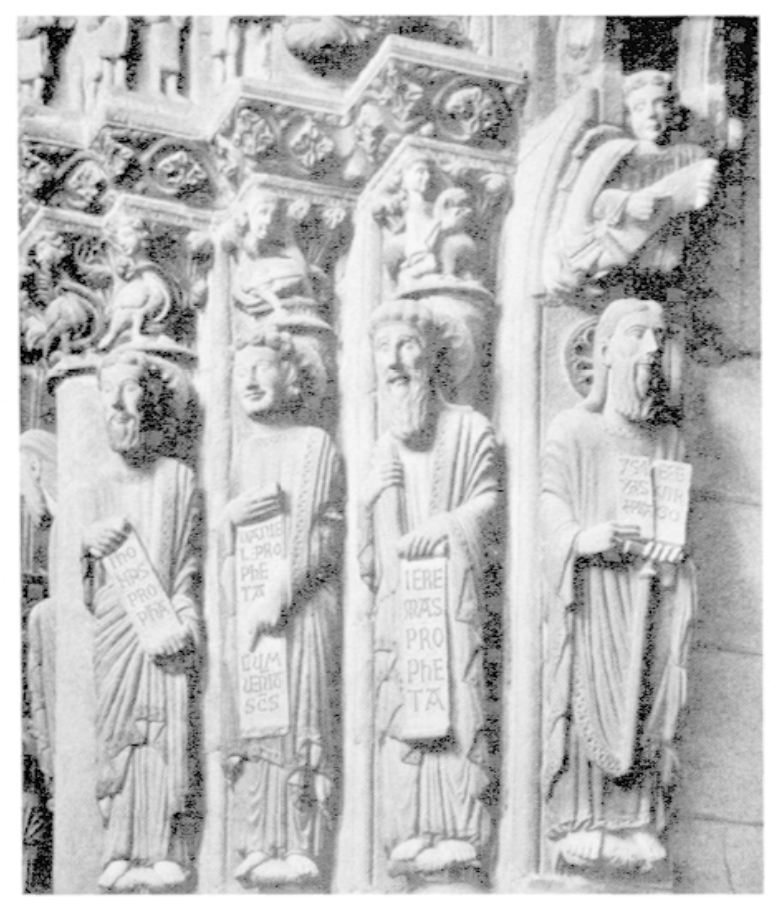

Hefe 6. - Porlail omest de la cathédrale d'orense. l'apres 1. h. Porter. Rememesque Scoltpure of the Pilgrimage Roads. Bostout. 1923. fing. 85.5.

cessabir mertio de Daniel, que l'on a sur la chasuble du var siecle de Saint-Blasien dans l’éveché de Comstance averégalement la cilalion elassique d’lsaie (II, 1/, sur sumbon de la cathedrale de

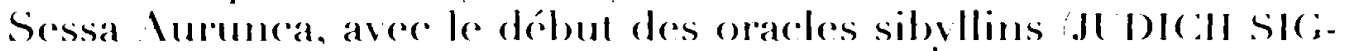
111 cilés par (2uodvuleleustio. (en la chapelle de San Pellegriono pres de Bominaeo Abrures fir. sur le reliquaire des lrois rois a la cathedrale de Colognerit. en la basilieque du Saint-Sépulere à

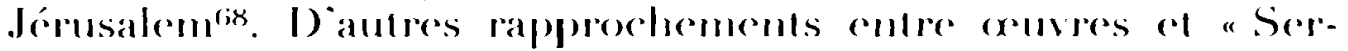
mon" soml possibles"6!).

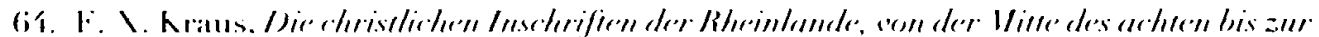

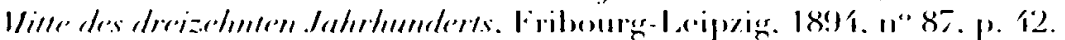

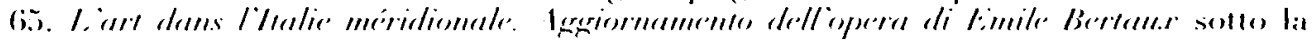

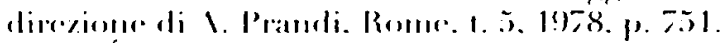

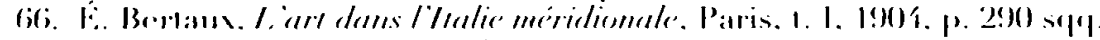

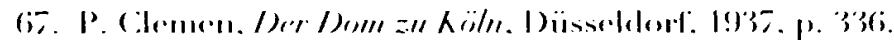

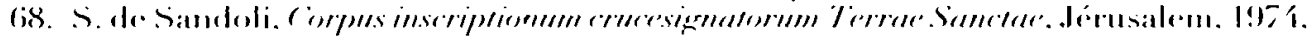
i" i2. p. (1) sq.

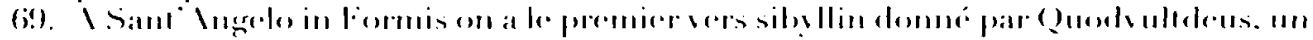

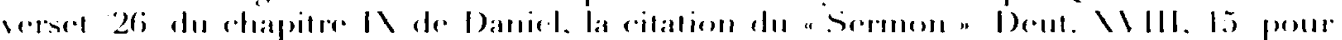

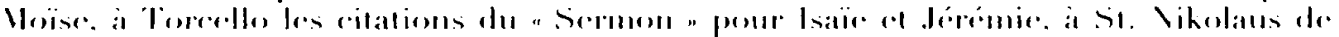

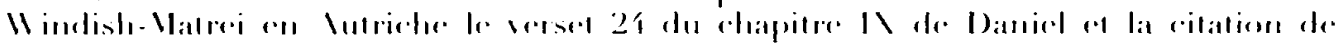




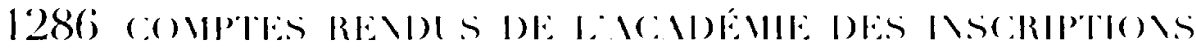

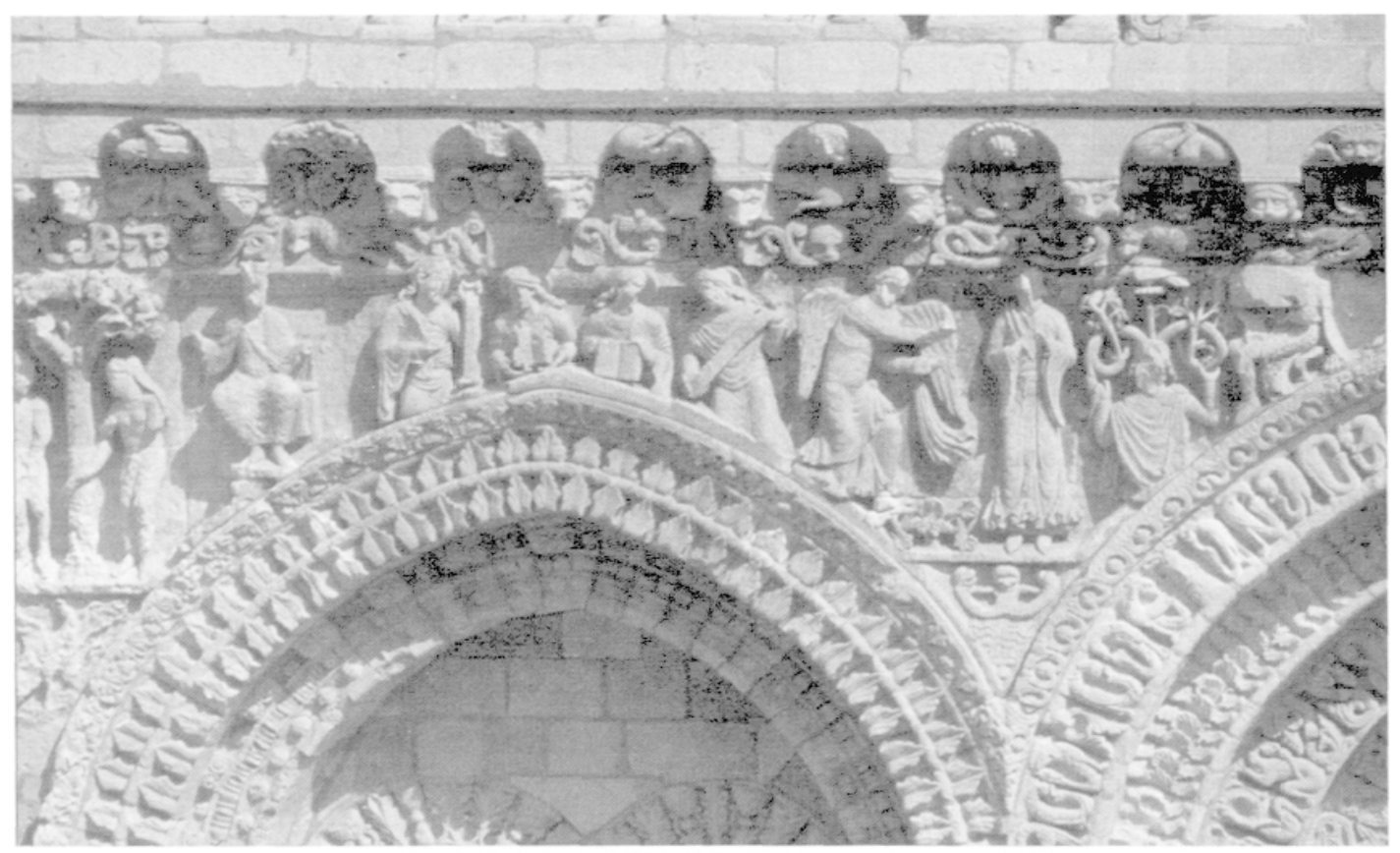

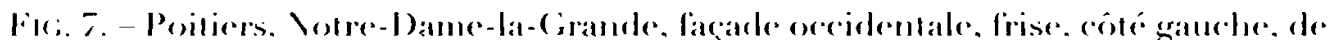

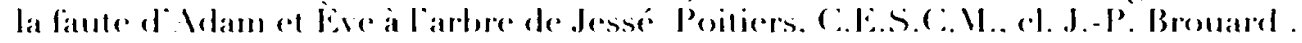

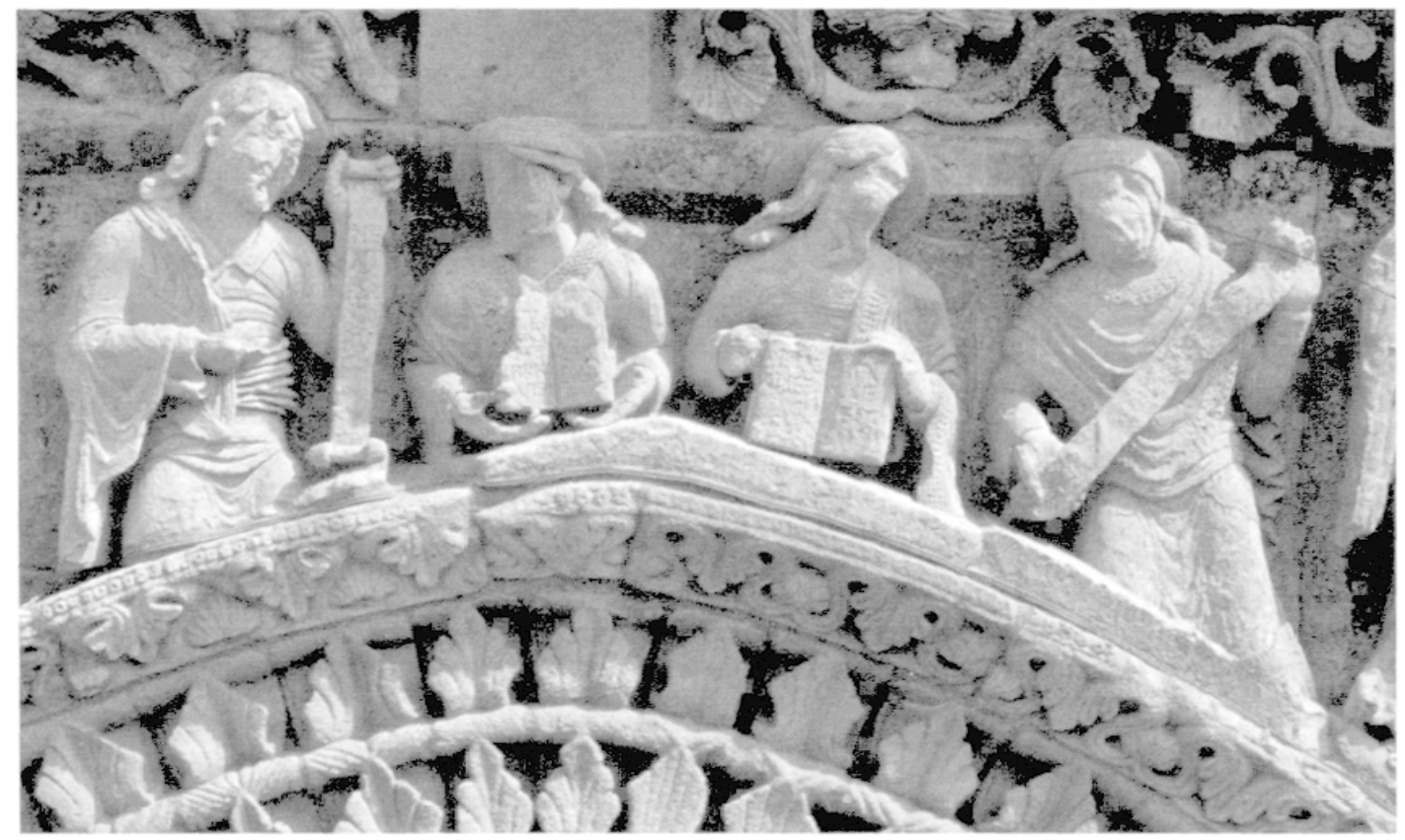

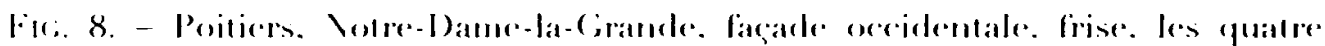

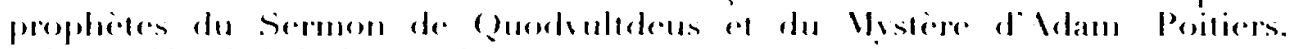
C..P.S.C..11.. c.l. J.-P. Bromard. 
Si l'on veut bien se souvenir de ce que tous ces exemples datent de la période la plus fournie des controverses judéo-chrétiennes, considérer que la citation de Daniel avec la "cessation de l'onction " ne peut s'adresser qu'à des Juifs - " alors cessera votre onction " est-il dit à Crémone et à Ferrare - et, que le choix de ces prophètes et de ces prophéties dérive d'un "Sermon contre les Juifs ${ }^{70}$, on devra se poser la question d'une influence directe des controverses judéo-chrétiennes sur ces programmes iconographiques et épigraphiques.

On peut en trouver confirmation dans les a disputes " et " dialogues " entre Juif et chrétien et dans les traités contre les Juifs. On s'y affronte en effet, à coup d'arguments scripturaires, tirés de l'Ancien Testament. Le verset 14 du chapitre VII d'Isaïe, Ecce virgo concipiel est un argument classique pour prouver l'Incarnation. On le trouve chez Tertullien, Maxime de Turin, l'Altercatio du $x^{*}$ siècle, Pierre Damien, Gilbert Crispin, Guibert de Nogent, Hildebert de Lavardin ${ }^{71}$, Richard de Saint-Victor ${ }^{72}$, le pseudoGuillaume de Champeaux, Pierre de Blois, Gautier de Châtillon, Alain de Lille, le traité anonyme du XIr" siècle. Certains auteurs citent aussi, ou à la place, le verset 1 du chapitre XI d'Isaïe, Egredietur virga, que retiendront le " Mystère d'Adam ", l'Altercatio du X' siècle, Guibert de Nogent, Pierre Alfonse, Pierre de Blois, Guillaume de Bourges. Citent Moïse avec Deutéronome, XVIII, 15: l'Altercatio du xe siècle, Pierre Damien, Gilbert Crispin, Rupert de Deutz, le pseudo-Guillaume de Champeaux, Pierre de Blois. Plus spécifique est la prophétie de Jérémie, qui est, curieusement tirée de Baruch, III, 36-38, un livre que ne reconnaissent pas les Juifs. C'est déjà le fait de Tertullien, et après lui de Maxime de 'Turin, Isidore de Séville de l'Altercatio du X' siècle, de Pierre Damien, Gilbert Crispin, Pierre le Vénérable, Gautier de Châtillon, Pierre de Blois, Alain de Lille, et du traité anonyme du XII" siècle. L'Altercatio précise que Baruch était le notaire de Jérémie, et Pierre de Blois commente : Nec murmuret Judaeus verba haec esse Baruch el non Jeremiae. Verba enim haec Baruch de ore

Quodvuludeus pour Moïse. Il faudrait y ajouter les cilations des prophètes dans les enluminures des manuscrits lat. 10133. Fol. 38, où, au registre supérieur, Sanuel cint David, au registre inféricur le personnage de gauche dit : DIC, SANCTE IDANIEI. I)IC:

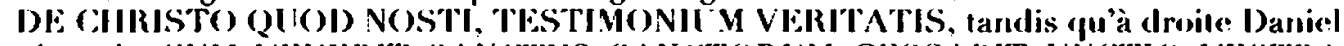

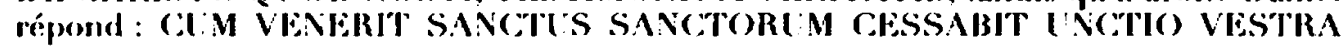
(C. M. Kauffimann, Romamesque Kamescripts 1066-1190, Iondres, 1975. p. 114 sq. (-1 ill. 255 ).

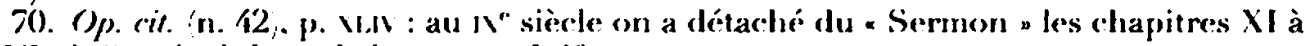
XVI où Quodvultdeus sadresse aux Juifs.

71. Sermon 101. Comura Judaess de Incrarnatione. dans Patrologie Iatine. 1. 171 c. 813.

72. Dans son De Eimmanuele libri duo. dans Patrologie latime, t. 196. (. 60). 


\section{COMPTES RENDUS DE L'ACADÉMIE DES INSCRIPTIONS}

Jeremiae accepit, sicut notarius illius, et scripsit ${ }^{73}$. Quant au chapitre IX de Daniel, sous la forme exacte et ungatur sanctus sanctorum, ou sous celle transformée de Quodvultdeus, cessabit unctio, a il est au centre de la controverse entre chrétiens et Juifs. Les exégètes chrétiens y voient une annonce très précise de la venue et de la Passion du Christ et de la destruction du Temple de Jérusalem, thèse que les Juifs réfutent $\star^{74}$. Au début du $\|^{\mathrm{e}}$ siècle Tertullien cite et commente longuement le passage de Daniel, puis, le reprenant à propos de la mort du Christ, en conclut : ut Daniel prophetavit, dicit enim : exterminabitur unctio. Paul Alvare de Cordoue $^{75}$, Isidore de Séville l'Altercatio du $x^{c}$ siècle, Fulbert de Chartres, Pierre Damien (cessabit unctio), Samuel dit du Maroc, Gilbert Crispin (cessavit unctio), Guibert de Nogent, Pierre Alfonse, Pierre le Vénérable, le pseudo-Guillaume de Champeaux (cessabit unctio vestra $)^{76}$, Gautier de Châtillon, Alain de Lille, le traité anonyme du XII" siècle, Guillaume de Bourges, Matfré Ermengaud $^{77}$ l'emploient à leur tour. Gilbert Dahan indique également que ce passage est cité par les auteurs juifs, Joseph Qimhi dans Sefer ha-berit (Le Livre de l'Alliance) et Joseph Ben Nathan l'Official de Sens, dans Sefer Yosef ha-meqaneh (Ie Livre de Joseph le Zélateur). Le lien entre ces œuvres et le « Sermon » de Quodvultdeus peut être des plus évidents : l'Altercatio du X' siècle et Gilbert Crispin citent les textes du Sermon pour ses quatre premiers témoins, Isaïe, Jérémie, Daniel et Moïse, et le « traité anonyme contre les Juifs » du xu* siècle les textes d'Isaie, Jérémie, Daniel ainsi que celui de Virgile et les oracles sibyllins, Pierre le Vénérable cite les textes de Jérémie, Daniel, David, Habacuc selon le “Sermon " et évoque Nabuchodonosor et la sibylle ${ }^{78}$. L'Église d'Orient a connu ces mêmes controverses et a dialogues " - Origène, saint Justin... - avec de semblables références scripturaires : Jean Chrysostome, Eusèbe de Séleucie, discutent longuement « contre les Juifs» du chapitre IX de Daniel79, et la “Dispute

73. Patrologie latine, t. 207, 839. Cf. Jérímie, XXXVI, 4 : Scripsit Baruch ex ore Jeremiae.

74. Guillaume de Bourges, op. cit. (n. 13), p. 152, n. 1.

75. Patrologie latine, t. 2, c. 612-616, et 634 ('Tertullien) ; t. 121, c. $481-483$ (Paul Alvare).

76. Patrologie latine, t. 163, c. 1056: Cum venerit Sanctus sanctorum cessabit unctio vestra. Itaque vestra unctio tota cessavit dum Sanctus sanctorum venit, quia templo et regno caretis et in universo mundo dispersi et exscecrabiles estis, qui Messiam suscipere recusastis.

77. B. Blumenkranz, art. cit. (n. 41), p. 305.

78. Petri Venerabilis, Adversus Judaeorum inveteratam duritiem, Y. Friedman éd. (Corpus christianorum. Continuatio mediaevalis, 58), Turnhout, 1985.

79. Patrologie grecque, Origène, Contre Celse (c colloque - avec: un Juif), t. 11, c. 743-744 ; Justin, Dialogue avec le Juif Tryphon, ibid., t. 6, c. 471 et suiv. ; ibid., t. 48, c. 898-899, t. 85, c. 399.123 (tout le * sermon contre les Juifs au sujel de la venue du Sauveur $\bullet$ ). 
contre les Juifs " du IX ${ }^{e}$ siècle mise sous le nom d'Anastase le Sinaïte, présente les témoignages d'Isaïe, de Jérémie, de Daniel et de Moïse avec les mêmes références scripturaires que le "Sermon" de Quodvultedeus ${ }^{80}$. Il faut tenir compte de la vitalité du monde juif entre la fin du XI" et le début du XII'* siècle, et des controverses qu'a eues à cette époque l'Église chrétienne avec eux et avec les premiers hérétiques en Occident, avant la période des répressions et exilss". C'est alors qu'on a utilisé le "Sermon" attribué à Augustin, et conservé dans la liturgie de Noël, pour le "Drame des prophètes " puis le "Mystère d'Adam ", en relation évidente avec la polémique antijuive, "Sermon " dans lequel on avait précisément isolé au IX" siècle les chapitres XI à XVI qui s'adressaient aux Juifs. Si on a utilisé ce même "Sermon ", probablement à partir du drame religieux, au cours de cette même période de la fin du XI' au milieu du XIII'e siècle, c'est évidemment en lien avec les controverses de l'époque entre Juifs et chrétiens. Les inscriptions qui accompagnent un nombre important d'ceuvres, illustrant les témoignages vétérotestamentaires à l'encontre des Juifs conduisent à introduire les rapports conflictuels Juifs-chrétiens dans l'explication de ces programmes iconographiques.

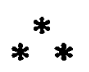

"La loi hébraïque prend fin lorsque cesse l'onction royale" affirme Étienne sur la table d'or de la cathédrale de Sens, qui oppose aussi la "Loi " qu'observait Saul à la grâce qui animera

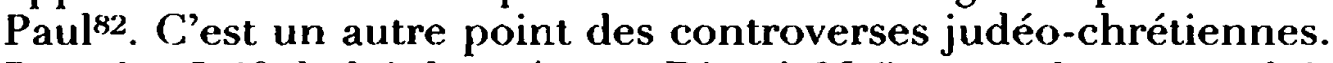
Pour les Juifs la loi donnée par Dieu à Moïse est « bonne et doit être observée "(Gilbert Crispin), pour les chrétiens la Loi est renouvelée par l'Évangile, la Synagogue a fait place à l'Église. Les inscriptions, là encore, viennent à l'appui de ce qui est dit dans les controverses judéo-chrétiennes.

80. Hid., t. 89, c. 1219, 1227 et 1239.

81. R. Manselli, - la polémique contre les Juifs dans la polénnique antihérétique •. dans Juifs et judaïme du languedoc (n. 38), p. $256 \mathrm{sq}$; A. (iraboïs, * lecoles et st ructures

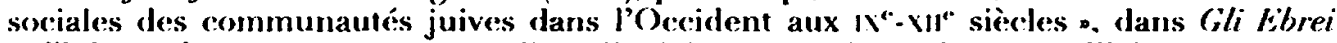
nell'aloo medioevo, t. II (Seltimane di studio del cent ro italiano di studi sull'alto medioevo. XXVI). Spolìte, 1980, p. 937-964 (Troyes, 1(660-1160, avec Rashi et ses fils ; l'ltalie) ; La

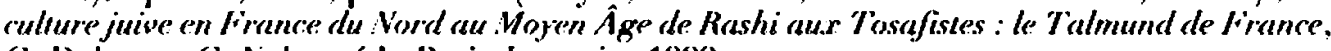
6. Dahan el (;. Nahon idl., Paris-L couvain, 1990).

82. Lex per hoysem datu est, gratict et veritus per Jesum ('hristum facta est (Jean. I, 17). Cf. un médaillon de I a Sauve-Majeure en 1231 avec la figure de Paul : QIII PRIMO SAIII IS

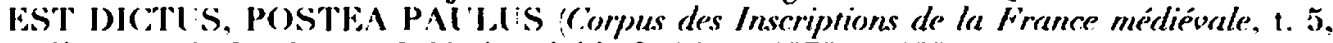
R. Favreau, B. Ieplant et J. Michaud ed.. Poitiers, 1979, p. 130). 
Déjà la célèbre mosaïque du début du ve siècle à Sainte-Sabine de Rome mettait en parallèle Pierre et l'Église de la circoncision (ECCLESIA EX CIRCUMCISIONE), avec Paul et l'Église des Gentils (ECCLESIA EX GENTIBUS). C'est dans le même esprit qu'au cul de four de San Silvestro à Tivoli sont présentés Paul à la droite du Christ - MICHI VIVERE CRISTUS EST ET MORI LUCRUM (Philippiens I, 24) -, et Pierre à sa gauche - DOMINUS LEGEM DAT ${ }^{83}$. On reprend bien à San Pietro in Civate, à propos de la Présentation au Temple, la parole du Christ : Je ne suis pas venu abolir la Loi, mais l'accomplir (Mt, V, 17) :

\section{SUPPLEAT UT VETEREM NON VENIT SOLVERE LEGEM ${ }^{84}$}

mais de façon plus générale on indique que par le Christ a la loi est renouvelée »-LEX RENOVATUR à la basilique du Saint-Sépulcre de Jérusalem ${ }^{85}$-, ce qui était dans l'ombre est révélé, comme le disent de belles inscriptions de Saint-Denis et d'Arles. Sur un des vitraux de son abbatiale de Saint-Denis Suger avait fait inscrire :

\section{LEGE DATA MOYSI JUVAT ILLAM GRATIA CHRISTI}

GRATIA VIVIFICAT, LITTERA MORTIFICAT.

"La Loi ayant été donnée à Moïse, la grâce du Christ la fortifie.

La grâce donne la vie, la lettre tue ».

TOLLIS AGENDO MOI.AM DF FURFURE, PAULE, FARINAM

MOSAICAE LEGIS INTIMA NOTA FACIS.

FIT DE TOT GRANIS VERUS SINE FURFURE PANIS

PERPETUUSQUE CIBUS NOSTER ET ANGELICUS.

a En tournant la meule, Paul, tu sépares la farine du son.

De la loi de Moïse tu révèles la connaissance profonde.

De tant de grains est fait le vrai pain non mêlé de son

Notre nourriture éternelle est angélique .

QUOD MOYSES VELAT CHRISTI DOCTRINA REVEIAAT

DENUDANT LEGEM QUI SPOLIANT MOYSEN.

* Ce que Moïse voile, la doctrine du Christ le dévoile.

Ceux qui dépouillent Moïse révèlent la Loi. n

83. H. I anz, Die romanischen Wandmalereien von San Silvestro in Tivoli : Ëin römisches Apsisprogramm der Zeit Innozenz III (Europaische Hochschulschriften Reihe, 28. Kunstgeschichte, 22), Berne-Francfort-New York, 1983. On observera que dans ces peintures sont représentés douze prophètes dont Isaie avec le texte du chapitre 7 , verset 14, et llabacuc avec le verset 2 de son chapitre 3, comme dans le Sermon de Quodvultdeus.

84. A. K. Porter, op. cit. (n. 53), III, 1917, p. 401.

85. S. de Sandoli, op. cit. (n. 68), p. 15. 
Par ceux qui dépouillent Moïse il faut entendre ceux qui enlèvent le voile qui couvrait sur le vitrail le visage de Moïse ${ }^{86}$.

A ces beaux textes composés par l'abbé Suger fait écho, une trentaine d'années plus tard, au grand portail de Saint-Trophime d'Arles, la statue de saint Paul, dont le phylactère porte deux vers léonins :

\section{LEX MOISI CELAT QUOD PAULI SERMO REVELAT} NUNC DATA GRANA SINAÏ PER EUM SUNT FACTA FARINA * La loi de Moïse cèle ce que la parole de Paul révèle.

Ainsi les grains donnés au Sinai sont-ils par lui transformés en farine ${ }^{87}$.

Dans la littérature antijuive de nombreux textes viennent corroborer cette fin de l'Ancienne Loi, figure ou ombre de la Nouvelle Loi : a Le Christ n'a pas rejeté la Loi en discutant, mais l'a changée en l'accomplissant " dit Augustin dans son Traité contre les Juifs ${ }^{88}$. Dans le Nouveau Testament est accompli ce qui était figuré dans la Loi écrit Raban Maur dans son Livre contre les Juifs ${ }^{89}$. Il faut casser la coque (la Loi de Moïse) pour arriver au noyau (la Loi du Christ), explique le pseudo-Guillaume de Champeaux ${ }^{90}$. "La vérité arrivant, l'ombre cesse " commente Alain de Lille ${ }^{91}$.

Toutefois il est clair qu'on a, plus souvent, insisté sur l'intransigeance des Juifs - SERVANT JUDAEI DURITIAM FIDEI lisait-on dans la basilique du Saint-Sépulcre de Jérusalem ${ }^{92}$ - et sur leur aveuglement. La venue du Christ, c'est la fin de la Loi mosäque, c'est la chute de la Synagogue à qui succède l'Église. Sur les bords de l'autel portatif de Stavelot, œeuvre d'environ 1160, aux musées royaux d'Ârt et d'Histoire de Bruxelles, on peut lire :

\section{QUAM COLIT ECCLESIA CRUX MORS VICTORIA CHRISTI PER SANCTOS PATRES PATRIARCHAS ATQUE PROPHE- TAS}

86. Suger, Kiuores, t. I, éd. et trad. F. Ciasparri, Paris, 1996 (les Classiques de l'H tistoire: de France: au Moyen $\hat{A}$ gej, p. 1/48-151, 235 et $238 \mathrm{sq}$. On notera que dans le vitrail de l'Arbre de Jessé Moise et Isaije sont accompagnés des lextes du sermon de Quodvultdeus et qu'y figure aussi Balaal avec le texte que lui donne le Mystère d'Adam o. Au vitrail de: l'arbre de Jessé de la cathédrale de Chartres Mö̈se est le seul avec: un verset sur sa banderole: : SISSCITABIT DEL iS VOBIS qui est aussi le texle: du * Sermon *.

87. Corpus des /ruscriprions de la France médiévale, 1. 14, R. Favreau, J. Michaud e: B. Mora éd., Paris, 1989, p. 14 sq.

88. Patrologie latine, t. 12, s. 53.

89. Thesaurus novus anectotorum, E. Martène et L. Durand éd., Paris, 1989, p. 44 sq.

90. Patrologie latine, 1. 163, c. 104.5.

91. Hid., t. 210, c. 10. (Gilbert Crispin parle aussi de voile figurarum oelamine adumbrata, éd. citée, p. 14j. (.f. Colossiens. Il, 17 : umbra futurorum.

92. S. de Sandoli. op. cit. (n. 68 ; , p. 35. n" 44 . 
ANTE FIGURATA FUIT ET PRAESIGNIFICATA ET TAMEN HEC CECA NUNDUM CREDIT SYNAGOGA * La croix que vénère l'Église, mort et victoire du Christ, a été préfigurée et présignifiée par les saints pères, patriarches et prophètes, et cependant cette synagogue se refuse encore à croire $\$^{93}$.

Sur le dessus de l'autel et en son centre l'Église (ECCLESIA) et la Synagogue (SINAGOGA) encadrent un triple SANCTUS (fig. 9). Sur la table d'un autel portatif, de même date, en l'église Saint-Vit de Mönchen-Gladbach la Crucifixion est représentée entre l'Église et la Synagogue, avec le commentaire suivant :

\section{GAUDEAT ECCLESIA DIRA DE MORTE REDEMPTA}

LEGIS SUMMA PERIT DUM MUNDUM VITA REDEMIT

* Que l'Église, rachetée d'une mort cruelle, se réjouisse.

La somme de la Loi périt tandis que la Vie a racheté le monde ${ }^{94}$.

Toujours des environs de 1160 une couverture au trésor de la cathédrale de Trèves représente la Crucifixion, avec, de gauche à droite, Marie, l'Église, le Crucifié, la Synagogue, saint Jean, et le commentaire de chacun des personnages :

+ ISTA FLET, HEC SURGET, OBIIT HIC, CADET HEC, DOLET ISTE

a Celle-ci pleure, celle-ci se lève, celui-ci meurt, celle-ci tombe, celui-ci se lamente ${ }^{95}$,

et un émail champlevé rhénan du xı'e siècle au musée du Louvre à Paris a exactement la même disposition, avec le même type de commentaire :

\% HEC PARIT (Marie), HEC CREDIT (l'Église), OBIT HIC (le Christ),

FUGIT HEC (la Synagogue), HIC OBEDIT (Jean) ${ }^{96}$.

93. M.-M. Gauthier, op. cit. (n. 34), 1972, n०95, p. 351 sq. ; Die \%eil der Staufer. (ieschichte. Kunst. Kultur, R. Haussherr dir., t. I, Stuttgart, 1977, p. 409, ill. 336; Rhin-Meuse. Art et civilisation 800-1400, Bruxelles-Cologne, 1972, p. 252 ("...Christum negant Deum esse, et quod eum nundum venisse dicunt $\bullet$, dit Fulbert de Chartres, Patrologie latine, t. 141, c. 308).

94. M.-M. Gauthier, op. cit. (n. 3/4), n'100, p. 354.

95. H. Schnityler, Rheinische Schatzkammer. Die Romanik, Düsseldorf, 1959, fig. 9 et n०3, p. 14.

96. P. Thoby, Le Crucifix, des origines au concile de Trente. Fitude iconographique, Nantes, 1959, pl. I.XI. 


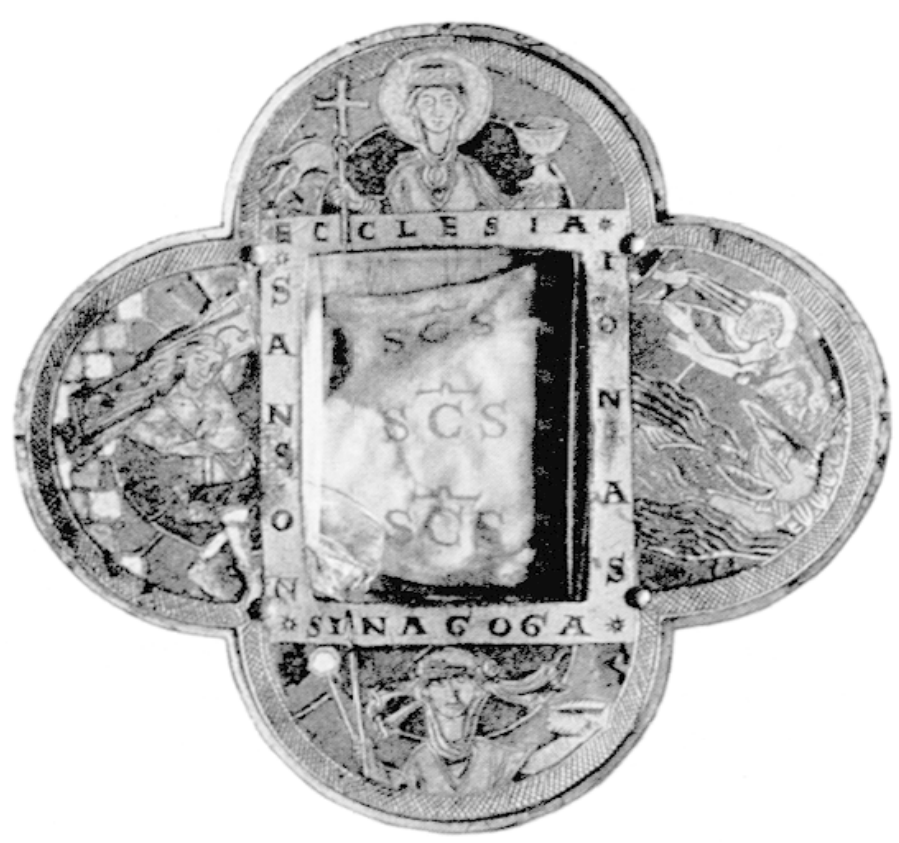

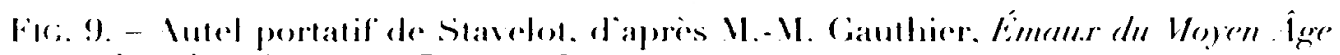
occidemul. Fibourur. 1972. p. 13\%.

Sur la châsse de saint Éleuthere a la cathédrale de Toumai de la premioner moitie du ville siecle, on lit:

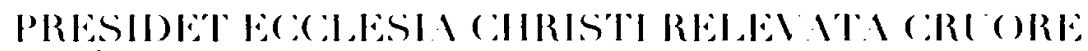

"L"Église préside. rachelée par le sang du Christ"

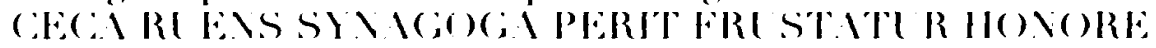

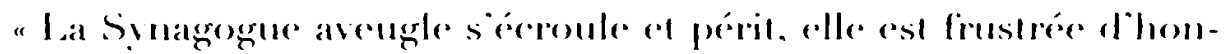
neorr

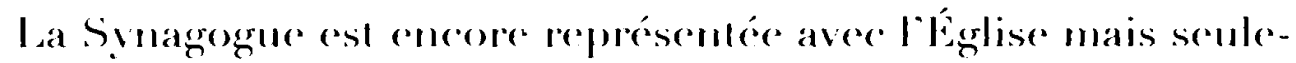
ment identifié par une inseriplion sur un autel portatif du milien

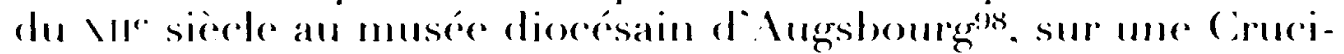
fixion en ivoire du muséce de Berlin-Dalhems?" sur une eroix

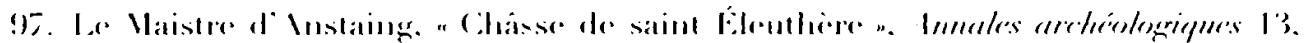
$185 \%$. 1. 11\%-12 i.

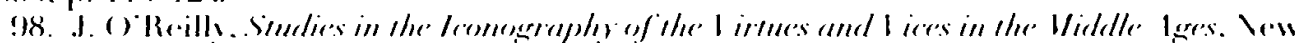
Tork-1 conderes igs8. pl. '3(ial.

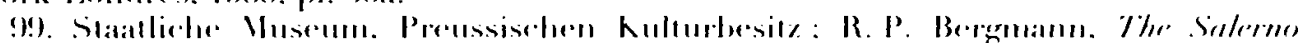

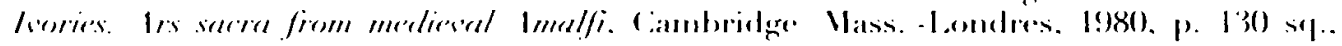
lige listi. 


\section{COMPTES REVDUS DE L'ACADÉMIE DES INSCRIPTIONS}

mosane en cristal de roche d'environ 1170 aux Musées royaux de Bruxelles ${ }^{100}$, sur un émail champlevé du milieu du XII* siècle à Gault-en-Forêt et sur un vitrail de la cathédrale de Bourges ${ }^{101}$. La croix de dame Gunhild à Copenhague, vers 1075, a l'Église à la droite du Christ (ECCLESIA SANCTA), à sa gauche la Synagogue (SYNAGOGA), en haut : VITA, en bas MORS ${ }^{102}$. C'est bien le sens général : la Synagogue tombe, s'écroule, fuit, meurt, la Loi mosaïque périt ${ }^{103}$, a Aux fidèles du Christ la Loi est inutile „ disait déjà saint Justin ${ }^{104}$. " A toi est la Loi écrite qui condamne, dit la Disputatio Ecclesiae et Synagoguae de Gilbert, à moi est donné l'Évangile qui sauve ${ }^{105}$, œuvre qui met en scène la Mère qu'est la Synagogue et la Fille qu'est l'Église, avec l'interpellation répétée : mater caeca, mater surda et caeca, mater caeca et misera. L'aveuglement des Juifs est d'ailleurs un leitmotiv de tous ces ouvrages ${ }^{106}$. Plusieurs de ces traités ou disputes sont cependant le fait de Juifs convertis. On signalera deux exemples épigraphiques de Juifs convertis, l'un par saint Austremoine ${ }^{107}$, l'autre par saint Nicolas ${ }^{108}$, dans les vitraux du XIII $^{\mathrm{e}}$ siècle aux cathédrales de Clermont et d'Auxerre.

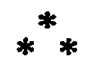

Dans la partie centrale de la table d'or de la cathédrale de Sens, point de départ de cette étude, est représentée l'image on ne peut plus classique du Christ de l'Apocalypse, trônant

100. H. R. Hahnloser, S. Brugger-Koch, Corpus der Ilartseinschliffe des 12-15. Jahrhunderts, Berlin, 1985, n"53, p. 96 et pl. coul. VIII.

101. A. Boinet, dans Bulletin de la Société nationale des Antiquaires de France. 1907 , p. $142-146$

102. J. Beckwith, Ivory Carvings in Early Medieval Fingland, Londres, 1972, p. 127, n"13, fig. 82-83; A. Goldschmidt, Die Filfenbeinskulpturen aus der nomanischen \%eit, XI.-XIII. Jahrhundert, t. III, Berlin, 1972, p. 35 sq., pl. 13-44.

103. On peut voir aussi sur la Bible de I ambeth à I ondres (Lambeth Palace library, ms. 3, fol. 307). Figlise et Synagogue, cette dernière avec le commentaire : I.FX PETIT OCCASUM. Dans les rapports entre Juifs et iconographie, la Synagogue a toujours retenu particulièrement l'attention des chercheurs, ainsi de B. Blumenkranz, op. cit. 'n. 1); II. I iebeschütz, Synagogue und ecclesia..., Heidelberg, 1983.

104. Patrologie latine, t. 6, c. 538.

105. Thesaurus novus anecdotorum, E. Martène et U. Durand éd., I . V, Paris, 1717, c. 1501 et passim.

106. Patrologie latine, t. 116, c. 167 ; t. 149, c. 351 (avec un long passage chez. Samuel le Marocain sur l'abaissement de la Synagogue et l'élection de l'F́glise, c. 360-362, t. 189, c. 574 ; t. 207 , c. 860 ; t. 213 , c. 799 , et aussi Matfré Ermengaud).

107. Vita prima, Acta Sanctorum, Novembre 1, p. 51 sq. (Corpus des Inscriptions de la France médiévale, t. 18, p. 171).

108. J. de Varazze, legenda aurea, G. P. Maggioni éd., 2 éd., Florence, 1998, t. 1, p. 44 sq. (Corpus des Inscriptions de la France médiévale, t. 21, p. 25 sq.). 
entre des anges et les évangélistes. Il est " le roi des rois "(Ap XIX, 16), l'alpha et l'oméga (Ap I, 8 ; XXI, 6, XXII, 13), et on aurait bien pu en rester là. Pourtant on a pris soin de préciser qu'il n'était pas seulement le commencement et la fin, mais aussi « le commencement sans commencement ", emprunt à saint Augustin, et on a ajouté deux distiques, l'un sur la Trinité, l'autre sur la légitimité du culte des images, à nouveau deux des critiques constantes des Juifs. A Sens les vers sur la Trinité sont les suivants :

\section{SOLUS CUNCTA REGO TRINUS ET UNUS EGO TRINLS AB AETERNO DEUS IJNUS CUNCTA GUBERNO « Je dirige tout, seul, moi qui suis Trine et un ; Trine de toute éternité, Dieu un, je gouverne toutes choses ".}

On peut rapprocher ces textes d'une série d'autres inscriptions du XII" ou XII" siècle : TEMPORA TRINA REGO TRINUS ET UNUS EGO (S. Cyriacus de Neuhausen près de Worms), ... DEUS IDEM CUNCTA GUbernans (Sahl, Danemark), SOLUS AB ETERNo CREO CUNCTA CREATA GUBERNO (châsse de saint Remacle à Stavelot et châsse de Notre-Dame au trésor de la cathédrale d'Aix-la-Chapelle), CUNCTA CREATA REGO, TRINUS ET UNUS EGO (moule à hosties du musée national du Moyen Âge à Paris ${ }^{109}$. La question de la Trinité esl abordée darıs un grand nombre de traités contre les Juifs ou des "disputes ": Évagre, le pseudoIldefonse, Raban Maur, l'Altercatio du X' siècle, Fulbert de Chartres, Pierre Damien, Gilbert Crispin, Guibert de Nogent, Pierre Alfonse, Gilbert (mi-xIles.), le pseudo-Guillaume de Champeaux, Pierre de Blois, Gautier Châtillon, Alain de Lille, le traité anonyme du XII" siècle, Guillaume de Bourges. Les arguments chrétiens sont toujours les mêmes. Dieu dit faciamus hominem ad imaginem et similitudinem nostram (Genèse I, 26), parce qu'il y a un pluriel (faciamus), et un singulier (imaginem), l'apparition de trois hommes à Abraham à Mambré (Genèse, XVII, 2-3), qui les salue en disant, au singulier : "Mon Seigneur ", enfin la triple acclamation Sanctus, sanctus, sanctus d'Isaie (VI, 3). Lors de représentation iconographique de ces trois passages - c'est le cas du triple sanctus dans l'autel de Stavelot déjà cité -, on peut, aussi, mais de façon moins évidente que pour le "Sermon " de Quodvultdeus, penser à une influence des controverses entre Juifs et chrétiens.

16.). Sur les inscriptions relatives à la Trinité, woir R. Favreau. * Épigraphie el thécologie . dans fipigraphie ef icomegrophie. R. Favreau dir. 'Civilisation médiévale, II; Poitiers, 1996. p. 37-49. 


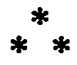

a Il remplit tous les lieux mais cependant n'est pas lui-même localisable

lui qu'une peinture superficielle indique être en ce lieu. »

Par ce distique les auteurs du programme de la table d'or de Sens veulent signifier que le Christ représenté trônant en majesté est bien Dieu, mais que l'image que l'on a sous les yeux n'est qu'une «peinture superficielle ». Une série d'inscriptions du même genre peut être réunie pour le XII" et le XIII' siècle, c'est-àdire à une époque où le culte des images n'est pas remis en cause par les chrétiens. La validité en est même confirmée à cette époque par le Décret de Gratien.

Au tympan de San Miguel d'Estella le Christ est représenté en majesté, dans une mandorle quadrilobée, entouré des symboles des évangélistes, comme à Sens. Sur la mandorle on a inscrit :

NEC DEUS EST NEC HOMO PRESENS QUAM CERNIS IMAGO SET DEUS EST ET HOMO QUEM SACRA FIGURAT IMAGO

« Cette présente image que tu vois n'est ni Dieu ni homme, mais il est Dieu et homme celui que figure cette image sacrée .

Ces deux vers remarquablement frappés sont de Baudri de Bourgueil, et on les retrouve chez Herrade de Landsberg et, avec des variantes dans plusieurs œeuvres du xIle siècle :

- reliquaire phylactère à quatre lobes mosan, vers 1165 , au musée de l'Ermitage à Saint-Pétersbourg (mêmes vers qu'à Estella) (fig. 10) ;

- retable de Saint-Denis du temps de l'abbé Suger, sur les bords du quatre-feuilles entourant le Christ assis :

HIC DEUS EST ET HOMO QUEM PRESENS SIGNAT YMAGO ERGO ROGABIT HOMO QUEM SCULPTA FIGURAT YMAGO ;

- tympan de la Porta dei Mesi à la cathédrale de Ferrare :

NECDEUS EST NEC HOMO PRAESENS QUAM CERNIS IMAGO SET DEUS EST ET HOMO PRAESENS QUAM SIGNAT IMAGO ;

- monstrance en métal doré du début du XIII siècle :

HIC DEUS EST NEC HOMO QUEM PRESENS SIGNAT IMAGO SET DEUS EST ET HOMO QUEM SCULTA FIGURAT YMAGO ; 


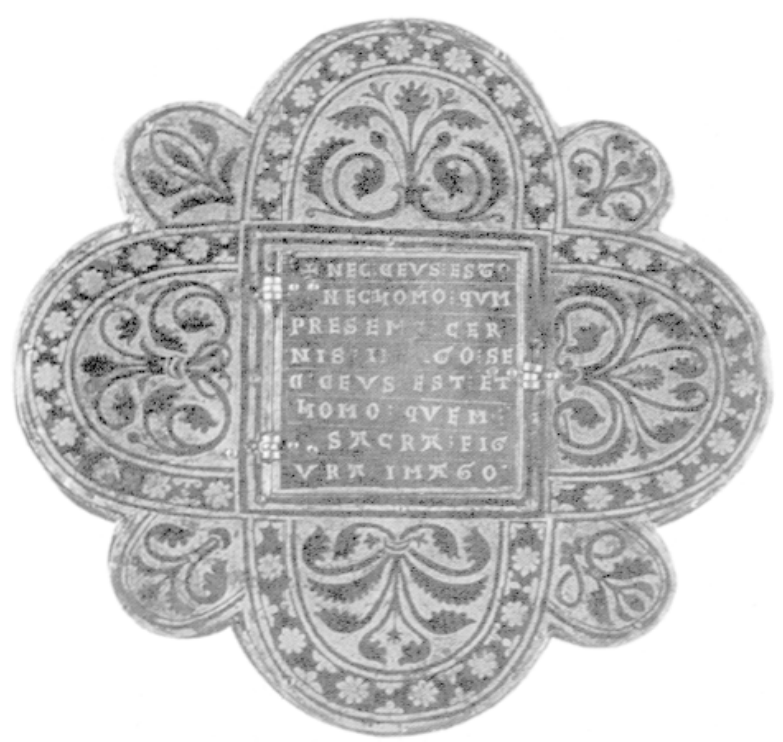

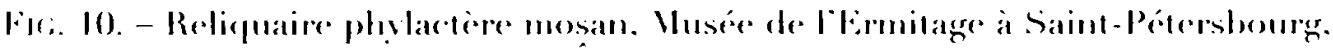

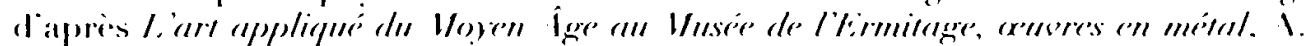

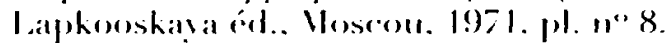

- ympan de Saint-Boniface à Ilameln l'm moilie du \II"s.,

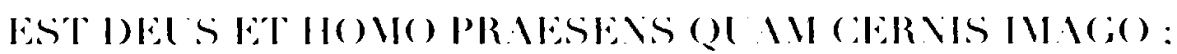

- mosä́que de la chapelle Saint-Clément à Saint-Marede lenise:

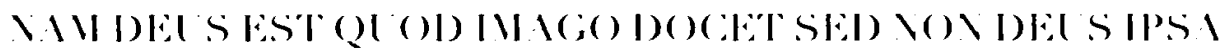

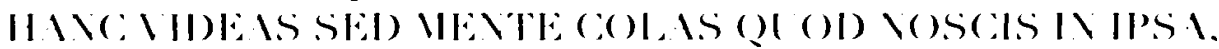

ce qui est more des formules dont Cinillanme de Mende reeommandera diuser pour expliefuer ee qüil faut entendere par la lemémalion des images:

- lable dautel en or de Saint-l ietor de Xanten 1129:

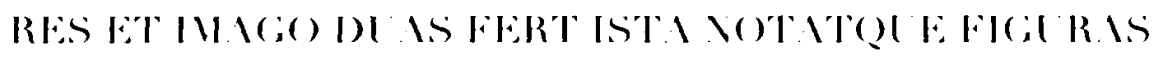

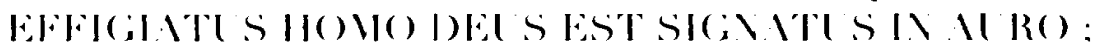

- ivoire du VIr" sierele au Vuseo mavionale à Forence :

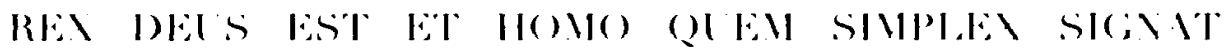
IIIT;()110.

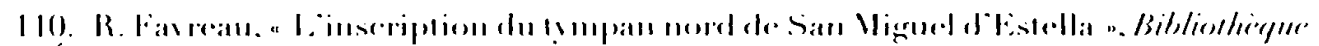

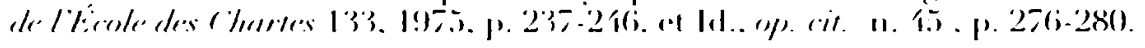


Les images, à cette époque, sont rejetées par les Juifs, les musulmans, et les hérétiques du XIIe siècle, de Pierre de Bruys aux cathares. Ce n'est pas aux musulmans - sauf peut-être à Estella ni sans doute tellement aux hérétiques - car les inscriptions citées ne sont pas du Midi de la France - que s'adressent ces mises en garde d'avoir à vénérer non les images mais ce qu'elles représentent. Et c'est encore aux Juifs que l'on peut penser, dans la mesure où, dans toutes les " disputes » de ce temps, ils rappellent aux chrétiens que Dieu a interdit de faire aucune image sculptée (Exode, XX, 4-5). Sans doute la question est-elle plus d'actualité à l'époque romane où la sculpture en ronde-bosse supplante le bas-relief. En tout cas, dans les controverses judéo-chrétiennes occidentales la critique des images par les Juifs n'apparaît qu'à la fin du $\mathrm{xl}^{\mathrm{e}}$ siècle dans la Disputatio de l'abbé de Westminster Gilbert Crispin. Le Juif s'étonne de voir les chrétiens représenter " Dieu misérable, pendant sur le gibet, fixé par des clous à la croix, ce qui est horrible même à voir, et de l'adorer... Il vous arrive de représenter Dieu assis sur un trône élevé et bénissant de sa main tendue, et autour de lui, comme pour souligner le prestige de sa dignité, un aigle et un homme, un veau et un lion. Les chrétiens sculptent les images, les fabriquent et les peignent, d'où et où ils peuvent, les adorent et vénèrent. Ce que la Loi donnée par Dieu interdit de toute manière ». Lc chréticn répond: « Nous faisons pour Dieu des peintures, des ciselures, des sculptures, mais nous ne les adorons pas ni ne leur réservons un culte divin. Car cette croix, que nous disons une croix sainte, nous disons que c'est du bois, non Dieu, et nous ne lui reconnaissons aucune vertu en elle-même ou d'elle-même. Mais après qu'elle a été consacrée par la bénédiction d'un pontife en mémoire de la Passion du Seigneur, nous l'adorons et la vénérons, non par un culte réservé à Dieu, mais avec la vénération qui lui est due. Le chrétien n'adore nulle image par un culte divin, mais il vénère et honore les effigies et peintures des choses sacrées par un culte d'honneur. „111

Le contemporain de Crispin, Guibert de Nogent, donne aussi la réplique à la critique des Juifs : a Nous adorons dans les signes visibles, les choses invisibles qui sont signifiées, bien plus nous conduisons l'esprit vagabond, par le regard soudain des peintures, comme par un avertissement, à se tourner vers les choses

111. The Works of Gilbert Crispin abbot of Westminster, op. cit. (n. 10), p. 50 sq. Avant Crispin Agobard de I yon indique sans s'étendre: ('hristianos idola asserunt (Judaei) adorare (Patrologie latine, t. 104, c. 88). 
intérieures... Sachez qu'à nous, à qui Dieu a donné la connaissance des lettres, sans faire appel à l'imagination et avec l'aide de la foi et de la contemplation, les sculptures ne sont pas nécessaires. Mais elles le sont pour ceux-là seuls qui, ignorants et sans finesse, apprennent par des aides de cette sorte, ce qu'il faut savoir, qu'ils ne peuvent atteindre par les lettres. ${ }^{112}$ Rupert de Deutz à son tour a répondu à l'accusation juive d'idolâtrie. Les arguments scripturaires sont un peu partout les mêmes : Dieu a ordonné de faire deux chérubins en or pour l'arche d'alliance (Exode, XXV, 18-20), il a prescrit à Moïse de faire un serpent d'airain (Nombres, XXI, 8-9), le décor du temple de Salomon comportait chérubins, mer d'airain reposant sur douze bœufs (1 Rois, VI, VII, VIII). \& Je fais une représentation du Christ, Dieu et homme, crucifié, et je l'adore, non par un vain espoir de santé et de salut, mais dans la foi et par la foi en celui qui, pour moi, selon cette représentation, a été pendu sur la croix. » Peut-être Rupert fait-il état de la vraie justification de l'image dans le christianisme, à savoir la possibilité de représenter Dieu à partir du moment où il a pris la nature humaine dans l'Incarnation, lorsqu'il écrit : " Dieu est invisible, et afin qu'il soit vu, il s'est fait homme (Deus invisibilis est, et ut videretur homo factus est) $\gg$. Un peu plus loin dans son texte on trouve un dialogue très proche des vers de Baudri de Bourgueil. « Le Juif : " Ce que tu adores n'est ni Dieu ni homme" (quod adoras nec Deus est, nec homo). Le chrétien : "Mais l'image figure l'un et l'autre " (sed utrumque figurat imago). „ Un peu plus loin le Juif note que le chrétien adore l'image du crucifix qui pour lui représente le Messie, c'est-à-dire le Christ, et ajoute : « Tu fais aussi d'innombrables images, tant masculines que féminines, et les temples sont pleins de représentations de ceux qui ne sont pas dieux. " Rupert fait répondre au chrétien : "C'est d'une manière louable que je sculpte de ciselures variées et je façonne sur toutes les parois des églises ; et je ne multiplie pas seulement les chérubins et les palmes, mais encore les peintures variées de la mémoire représentant les actes mémorables des saints, la foi des patriarches, la vérité des prophètes, la gloire des rois, la sainteté des apôtres, les victoires des martyres, et, parmi toutes leurs images sacrées, la croix qu'il convient d'adorer avec l'image de mon Sauveur brille pour mon admiration, parce que tu n'as pu le tuer par aucun genre de mort. ${ }^{113}$ Gautier de Châtillon évoque briè-

112. Patrologie latine. t. 156. (c. 524-525).

113. Ibidl, t. 170, c. $601-6988$. 


\section{COMPTES RENDUS DE L'ACADÉMIE DES INSCRIPTIONS}

vement l'accusation juive d'idolâtrie parce que les chrétiens vénèrent des crucifix et images des saints ${ }^{114}$.

Avec Alain de Lille (m. 1203) nous sommes à la fin du XII ${ }^{\mathrm{e}}$ siècle. La contestation des images ne vient plus seulement des Juifs, mais aussi des vaudois, des cathares - contre lesquels Alain s'employa -, voire des cisterciens à la suite de saint Bernard. Dans son De fide catholica contra haereticos sui temporis, praesertim Albigenses, libri quatuor, le premier livre est contre les cathares, le deuxième contre les vaudois, le troisième contre les Juifs, le quatrième contre les " païens ou mahométains *, et c'est dans ce dernier livre qu'il aborde la question du culte des images : a Les païens, avec les Juifs, nous insultent parce que nous avons des images et des sculptures dans nos églises... Si la loi mosaïue a interdit de faire quelque sculpture que ce soit, alors Moïse a péché, lui qui a fait les images des chérubins et les a installées dans le temple... On peut peindre les images des saints et l'image du Christ, afin que les hommes soient, par ce qu'ils voient, invités à se tourner vers l'invisible et que, à travers ce qui signifie, soit vénéré ce qui est signifié. Car, de même que les écrits sont les lettres des clercs, les peintures le sont pour les laïcs. ${ }^{115}$ Pour le reste Alain recopie textuellement Gilbert Crispin. Adam de Dore (?) dans son Pictor in carmine, écrit vers 1200 , reprend cette argumentation pratique, qui était déjà celle de la lettre de Grégoire le Grand à l'évêque de Marseille : les peintures notamment dans les églises cathédrales et baptismales, sont un peu "les livres des laics qui suggèrent aux simples ce qui touche le divin et qui excitent les lettrés à l'amour des écritures \$16. Guillaume de Bourges dit de même : « Aujourd'hui nous faisons des images, car de même que les écritures sont les lettres des clercs, de même les peintures sont les lettres des laïs... Nous ne croyons pas en les statues, mais nous adorons et mettons toute notre foi en la pierre d'angle qui, pour nous, alla en un tombeau de pierre afin d'ouvrir notre cœur de pierre. ${ }^{117}$

On pourrait retrouver la même argumentation dans l'église d'Orient à propos des Juifs. Non pas d'ordre théologique comme au temps de l'iconoclasme où l'on rejetait les images parce qu'elles ne représentaient que l'humanité du Christ, au risque de la trop distinguer de sa divinité (nestorianisme) ou de confondre les deux natures divine et humaine en une seule représentation

114. Ibid., t. 209 , c. 425 et 441 .

115. Ibid., t. 210 , c. $127-428$.

116. Guillaume de Bourges, op. cit. (n. 13), p. 35, note.

117. Ibid., p. 224 sq. 
(monophysisme). Ainsi Nicetas Stéthatos, moine du monastère de Stoudios au milieu du $\mathrm{Xl}^{*}$ siècle, écrit, dans un Traité contre les $J u i f s$, a accusant dès le début leur indocilité et leur infidélité : "...Adorez le Christ notre roi et notre Dieu, embrassez son empreinte sacrée dans son image. Car ce ne sont nullement, comme vous le dites, des idoles que nous adorons, nous qui croyons le Christ, ou mieux qui croyons au Christ. Autre chose, en effet, une idole, autre chose une image. Est idole ce qui n'a pas pour prototype, par exemple, un homme qui a vécu de notre existence et qui s'est montré juste dans les œuvres de justice au milieu des hommes, ce qui n'est composé que de matière, pierre ou bronze, que l'on sculpte entièrement en forme d'homme avec une poitrine, des genoux et tout l'ajustement des membres et des parties : c'est ce que nous appelons encore une statue, et celui qui la vénère et l'adore est vraiment idolâtre et adore la statue. L'image au contraire, peinte en couleur sur tableau ou mur, se rapporte à un homme, saint évidemment, qui a vécu dans celle existence avec justice et vertu, qui a brillé parmi les hommes par les cuvres des commandements de Dieu ou des miracles ou des prodiges, ou par la sagesse et l'enseignement de Dieu ou par la confession de la foi et la mort pour l'amour du Christ; si quelqu'un l'adore et la vénère, ce ne sont pas les couleurs ni la chaux, comme vous imaginez qu'il adore et embrasse, mais l'empreinte de celui dont la vie et la parole ont brillé plus que le soleil en cette vie : c'est à lui en tant que prototype, qu'est réservé l'honneur. "118 C'est reprendre saint Basile : "I' honneur rendu à l'image atteint le prototype. "119 On aura noté que le moine de Constantinople paraît écarter la statue au profit de la peinture, et peut-être préférer une représentation en buste plutôt qu'en pied. En 1158 ou 1163 Paschalis Romanus, à Constantinople, a abordé aussi la question dans sa Disputatio contra Judaeos. « I.e Juif interrogea : alors que Dieu a enseigné à ne pas adorer le bois, pourquoi, vous, chrétiens, le vénérez et adorez-vous, et en faites vous croix et images? Le chrétien : Est-ce que, en adorant la croix nous adorons la nature du bois? En rien, mais nous vénérons en lui le Crucifié... Lorsque j'adore l'image, je n'adore pas et n'honore pas la nature du bois, mais le Christ el ses saints... Lorsque j'adore l'image du Christ je dis : Gloire à toi, Seigneur, et non : Gloire à

118. Vicetas Stétathos, Opuscules of lettres, J. Darrouzès ćd. (Sources chótiennes. 81). Paris, 1961. p. 136i sq.

119. Patrologie grecque. 1. 32. (2. 149) (nam imaginis homor ad exempla iransit), el Sources chrétiemes 17. 190, repris par saint Jean I) amascène au vill" siècele (Patrologie gresque, 1. 94. De fide orthodora. I. 4. eh. 16. De sanrtorum imaginibus, c. 1167-1170). 
toi, bois ou peinture. Est-ce que lorsque j'adore la croix, je dis : Gloire à toi, bois ? Nullement. „120

Saint Thomas d'Aquin a abordé la question dans sa Somme théologique : « On ne doit aucune vénération à l'image du Christ en tant qu'elle est une chose comme du bois sculpté ou peint, parce qu'on ne doit de vénération qu'à la créature raisonnable. Il reste donc qu'on ne lui manifeste de la vénération seulement en tant qu'elle est une image... Le précepte en question (Exode, XX, 4) n'interdit pas de faire une sculpture ou une image, mais de le faire en vue de l'adorer... Il faut comprendre que l'adoration prohibée est celle des images que les païens fabriquaient pour vénérer leurs dieux. Quant à Dieu lui-même, puisqu'il est incorporel, aucune image de lui ne pouvait être proposée... Mais parce que, sous la nouvelle alliance, Dieu s'est fait homme, il peut être adoré sous son image corporelle. $121^{121}$ ne parle pas des Juifs, pas plus que, quelques années plus tard, Guillaume Durand dans son Rationale divinorum officiorum (livre I, Pictura et ornamenta), et certes le problème est de tous les temps. Mais c'est au XII' siècle que s'est intensifié le débat théologique entre Juifs et chrétiens, que ce sont multipliés les traités chrétiens et Juifs ${ }^{122}$, les « disputes » et " dialogues », et c'est à cette époque, et non à une autre, que l'on trouve cette série d'inscriptions pour distinguer l'image de ce qu'elle représente. On ne peut donc parler du “culte des images " aux $\mathrm{XI}^{\mathrm{e}}-\mathrm{XIII}^{\mathrm{e}}$ siècles en dehors du contexte des controverses judéochrétiennes, même si celles-ci ne sont pas seules en cause ${ }^{123}$.

Juifs et chrétiens s'opposent au Moyen Âge sur la caducité de loi mosaïque, la messianité de Jésus, l'Incarnation, avec de surcroît la naissance virginale, la Trinité, la Crucifixion, l'élection des Gentils et le rejet des Juifs, le culte des images ${ }^{124}$. On se gardera bien de voir une influence de ce débat chaque fois qu'il sera question de l'Incarnation, de la Crucifixion, de la Trinité, du culte des images. On ne pourra guère éviter de se poser la question quand on trouvera opposition de la Loi mosäque et de la Nouvelle Alliance, venue du Messie annoncée par les prophètes, déchéance de la Synagogue remplacée par l'Église. Les inscriptions qui

120. Paschalis Romanus, Disputatio contra Judeos, G. Dahan éd., dans Recherches augustiniennes, 11, 1976, p. 192 sq. et 203.

121. Somme théologique, t. 4, Paris, 1986, p. 197 sq. (III“ partie, Question 25, Article 3).

122. J.-Cl. Schmitt, * l'Occident, Nicée II et les images du vifl* au xiI" siècle *, dans Nicée II, 787-1987. Douze siècles d'images religieuses, Actes du colloque international Nicée II..., F. Boesflug et N. I cossky éd., Paris, 1987, p. 271-301.

123. B. Blumenkranz, op. cit. (n. 22), p. 285 : à propos du culte des images l'auteur dit que * la part prise par les juifs dans ce débat a été soit exagérée, soit par trop minimisée ».

124. Ibid., Troisième partie... La polémique judéo-chrétienne, p. 213-289. 
accompagnent nombre d'œuvres d'art aux X X ${ }^{e}$-XIII' siècles permettent de mieux cerner certaines des influences des controverses judéo-chrétiennes sur l'iconographie. D'autres plus compétents en matière d'histoire juive ou d'iconographie pourront sans nul doute préciser de nouveaux points. Mais il apparait que pour les $\mathrm{XI}^{\mathrm{e}}$-XIII' siècles en particulier, on ne peut faire l'économie de tenir compte de ces controverses lorsqu'on fait une recherche iconographique. C'est en tout cas la conclusion à laquelle mène l'examen d'un certain nombre d'inscriptions de cette époque.

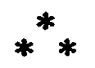

MM. Philippe Contamine, Michel Zink et Jean-Pierre Bablion interviennent après cette communication.

\section{LIVRE OFFERT}

M. François Chavot:x a la parole pour un hommage :

a J'ai l'honneur de déposer sur le bureau de l'Académie, de la part de son auteur, M'me Brigitte I,e Guen, un ouvrage intitulé Les associations de technites dionysiaques à l'époque hellénistique, Nancy, 2001, deux volumes in $-4^{\circ}$ de 355 et 224 p. Cette publication prend place dans la série des Études d'archéologie classique, que j'ai fondée en 1958 et que nos collègues de Nancy ont eu le mérite d'entretenir depuis lors en y accueillant des travaux de qualité. Notre correspondant Claude Brixhe veille activement à maintenir cette tradition, qui témoigne en faveur de la vitalité du centre de recherches nancéen, fort bien équipé et très actif. L'ouvrage de $M^{\text {me }}$ Le Guen a aussi bénéficié de l'appui du centre de recherches sur la Libye antique (Paris-Sorbonne), dont le directeur, le professeur André

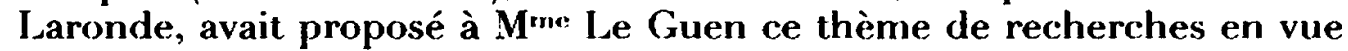
d'une thèse d'habilitation qu'elle a récemment soutenue.

C'est un grand sujet, qui touche à la fois à la religion, à l'économie et à la politique, dont les fêtes et les concours étaient constamment, dans les cités grecques, des manifestations révélatrices. A partir du début de l'époque hellénistique, acteurs, poètes, musiciens, danseurs s'organisent en associations professionnelles sous le patronage de Dionysos. Ces 